\title{
Navigating Molecular Evolution Using Substitution Mappings
}

\author{
by \\ Andrew Low, B.Sc. \\ A thesis submitted to the \\ Faculty of Graduate and Postdoctoral Affairs \\ in partial fulfillment of the requirements for the degree of
}

Master of Science in Biology with Specialization in Data Science

Ottawa-Carleton Institute for Biology

Department of Biology

Carleton University

Ottawa, Ontario

March 17, 2017 
(C)Copyright

Andrew Low, 2017 


\begin{abstract}
Since first proposed in 2002, Bayesian substitution mappings have found less use than might have been expected in the field of molecular evolution. Here, I first create a sequence simulator capable of generating true substitution mappings for simulated data under any time-reversible model in order to facilitate study of substitution mappings. I then investigate the utility of substitution mappings for two applications: detection of coevolving residues and scoring of amino acid substitution radicality to test the predictions of the nearly neutral theory. I find that mappings perform poorly for coevolution detection, but using mappings to find the radicality of an average amino acid substitution by scoring each observed substitution works well. Overall, substitution mappings look to be a potentially useful tool for some types of molecular evolution studies.
\end{abstract}




\section{Acknowledgments}

There are a lot of people to thank for this one. First up is Dr. Alex Wong, who successfully put the "super" in supervisor for the last 20 months. My committee members, Dr. Nicolas Rodrigue and Dr. Stephane Aris-Brosou, provided tons of helpful advice, and in Nic's case, computing resources that made my life significantly easier.

All members of the Wong Lab, both past and present, also deserve recognition for giving good feedback on all of my work in lab meetings.

Finally, thanks to my parents - who may not understand what it is that I'm researching, but sure are supportive of it regardless! 


\section{Table of Contents}

Abstract $\quad$ iii

Acknowledgments $\quad$ iv

Table of Contents $\quad$ v

List of Figures vii

1 Introduction 1

2 COMPASS 9

2.1 Abstract . . . . . . . . . . . . . . . . . 9

2.2 Introduction . . . . . . . . . . . . . . . . . . 10

2.3 Methods . . . . . . . . . . . . . . . . . . . . . . . 10

2.4 Verification ......................... 11

2.5 Example Usage: Coevolution Simulation . . . . . . . . . . . . . 12

3 Coevolution Detection $\quad 15$

3.1 Abstract . . . . . . . . . . . . . . . 15

3.2 Introduction . . . . . . . . . . . . . . . . . . . 15

3.3 Methods . . . . . . . . . . . . . . . . . . 18

3.3.1 Inferring Substitution Mappings . . . . . . . . . . . . 18 
3.3.2 Reading Mappings and Calculating MI . . . . . . . . . . . . . 18

3.3.3 Posterior Predictive P-Values . . . . . . . . . . . . . . . . . . 19

3.3.4 Data Simulation . . . . . . . . . . . . . . . 20

3.3.5 Metrics Used For Algorithm Evaluation . . . . . . . . . . . . . 20

3.4 Results and Discussion . . . . . . . . . . . . . . . . . . 21

4 Amino Acid Radicality 30

4.1 Abstract . . . . . . . . . . . . . . . . 30

4.2 Introduction . . . . . . . . . . . . . . . . . . 31

4.3 Methods .............................. 34

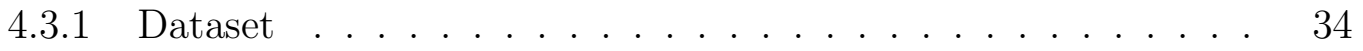

4.3.2 Substitution Mappings . . . . . . . . . . . . . . 35

4.3.3 Simulation of Data Under Mutation-Selection Models . . . . . 36

4.3.4 Correlations Between Body Size and Kr/Kc . . . . . . . . . . 37

4.3.5 Effects of Body Size on Rates of Nonsynonymous Substitution 37

4.4 Results . . . . . . . . . . . . . . . . . . . . . . 38

4.5 Discussion ........................... 44

5 Conclusions $\quad 49$

List of References $\quad 51$

Appendix A Details on Coevolution Simulation $\quad 61$

$\begin{array}{lll}\text { Appendix B COMPASS Verification } & 63\end{array}$ 


\section{List of Figures}

1.1 An example of a set of substitution mappings for a site, each representing a different draw from the posterior. For the site shown here, the ancestral state was cytosine, and all substitutions for each draw, including multiple changes along a branch, are shown. . . . . . . .

2.1 1A) Actual versus observed values of the $\mathrm{dN} / \mathrm{dS}$ ratio. As expected, at lower (more realistic) values, correlation is extremely tight, while the relationship is noisier at higher values of $\mathrm{dN} / \mathrm{dS}$. Tests were carried out with an GY94-style matrix, a 50 species tree, and 150 codons. 1B) Actual versus observed total branch length of trees input into COMPASS. COMPASS very accurately simulates the total tree length. Sequences were evolved under a GTR model with 1000 sites. 1C) Observed relative exchangeabilities for 130 sequences with 1000 sites under a GTR model (the sixth exchangeability was set to 1 and used to benchmark the others). Green squares represent the actual values. 100 replicates were carried out. 1D) Observed equilibrium frequencies for 130 sequences with 1000 sites, evolved under a GTR model. Green squares represent the actual values. 100 replicates were carried out. . 
3.1 A substitution mapping for two different sites. The site on the left is in state $\mathrm{A}$ for 46.1 percent of its evolutionary history and state $\mathrm{T}$ for 53.9 percent of its history. The site on the right is 54.5 percent $\mathrm{G}$ and 45.5 percent C. The joint probabilities for AG, TG, and TC are 46.1, 8.1 , and 45.5 , respectively. . . . . . . . . . . . .

3.2 PPV versus coevolution strength for a 130-species tree with no rate variation across sites. PPV is strong at all levels of coevolution strength

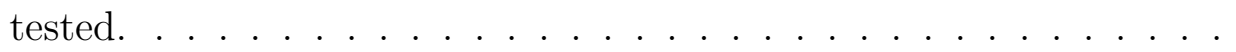

3.3 Effect of varying values of alpha on PPV for a 130-species tree. When rate variation across sites is high (low alpha values), PPV is poor. As rates across sites become more homogenous, performance sees substantial improvement, but still tends to underperform when compared to alignment MI with the MIp correction applied. . . . . . . . . . . .

3.4 Effect of contracting and elongating branches on PPV. Beginning with a 130-species tree with average branch lengths of 0.34 , branches were contracted to an average as short as 0.034 and elongated to up an average as long as 1.7. PPV values were low with both very short and very long branches, with optimal performance at average branch lengths between 0.3 and 0.7. At all branch lengths, phylogenetic MI performed worse than alignment MI with the MIp correction. . . . . .

3.5 Effect of alpha values on PPV within the optimal branch length range. As expected, performance tends to be poor when rate variation across sites is high, and improves as rates become more homogenous. . . . . 
3.6 A comparison of PPV for alignment-based MI with the MIp correction applied and phylogenetic MI on a 500-species tree with no rate variation across sites. Alignment-based MI outperforms phylogenetic MI at every level of coevolution strength tested. . . . . . . . . . . . .

3.7 Actual MI plotted against the MI inferred by PhyloBayes. Correlation among independent sites (in red) is very tight, while the amount of MI present between coevolving sites (shown in blue) is consistently underestimated. The dashed line shows a 1:1 correlation. . . . . . . . 28

4.1 The phylogeny used in the present study. Species considered large are found on red branches, while small-bodied species are in blue. . . . .

4.2 An example of a substitution history created by PhyloBayes. At the root of the clade, the site being examined is an alanine. Within the bat clade, an alanine to glycine substitution occurs, and receives a score of 1, while an alanine to phenylalanine substitution occurs on the branch leading to the panda, walrus and seal, and receives a score of -4 . . . .

4.3 Inferences of differences in substitution radicality from simulated datasets, with smaller population sizes in red and larger population sizes in blue. When population size difference is nonexistent (panel A) or small (panel B), there is no discernible difference in radicality between species. With larger population size differences of 100-fold (panel C) and 500-fold (panel D), small population-size species tend to have more radical substitutions. . . . . . . . . . . . . .

4.4 Radicality of average substitution for large vs. small-bodied species. More genes showed in large-bodied species had negative scores (radical substitutions) than small-bodied species. . . . . . . . . . . . . . 40 
4.5 Correlation coefficients for a relationship between body size and $\mathrm{Kr} / \mathrm{Kc}$ for the polarity-volume model. Far more genes than would be expected under a null model have positive correlation coefficients. . . . . . . . 42

4.6 Boxplot of $\omega$ for each gene in which the three- $\omega$ model was a significant improvement over the null model. Large-bodied species show a significantly higher average $\omega$ than small-bodied species. . . . . . . . 


\section{Chapter 1}

\section{Introduction}

Since the early 1960s, there has been interest in reconstructing DNA sequences of ancestral species in order to replay the tape of evolution and gain insight into how sequences and genomes evolve (Pauling et al., 1963). For example, in order to understand the origins of retrotransposons Adey et al. reconstructed the ancestor of the F-type subfamily of LINE-1 retrotransposons and found that the ancestor had promoter activity, shedding light on the origin of this family of retrotransposons (Adey et al., 1994). Similarly, an ancient ribonuclease was reconstructed and found to have a large change in behavior (an increase in catalytic activity against dsRNA), which supported the hypothesis that the protein had a different function in the ancestor in question before being converted to its new function (Jermann et al., 1995). More recently, ancestral reconstruction studies have elucidated the mechanism of action

of a cancer drug by reconstructing ancient kinases (Wilson et al., 2014), helped to understand how paralogs within a genome evolve different functions (Hudson et al., 2015), and tested hypotheses of adaptation in Drosophila melanogaster (Siddiq et al., 2017).

Early studies within the field tended to rely on maximum parsimony (MP) - the idea that the sequence of changes that produces the fewest total number of changes 
is likely to be the best one. However, there are many issues with using MP, both for inferring the underlying tree structure and reconstructing ancestral sequences. Notably, MP was generally used only with closely related species, as when used on species which were more diverged, several scenarios could be equally parsimonious. Due to the non-parametric nature of MP analysis, they would all be considered equally likely (Stewart, 1995). An additional trouble with MP lies in the fact that it considers all substitutions equally likely. In the case of DNA sequence reconstruction, this is obviously an incorrect assumption. Frequencies of the 4 nucleotides can be very different, and the ratio of transitions to transversions, as well exchangeabilities between nucleotides, are known to be far from equal, although these parameters can be accommodated using the Sankoff algorithm (Sankoff, 1975). For ancestral reconstructions in particular, the issue of compositional bias was shown to cause issues, making them less accurate that other approaches (Collins et al., 1994; Eyre-Walker, 1998). Additionally, trees with long branches where multiple substitutions might be expected will infer reconstructions that are less accurate than those inferred under other frameworks (Maddison, 1995; Zhang and Nei, 1997).

Due to the issues with reconstructions inferred using MP, new methodologies were developed. The first major improvement to ancestral reconstruction to appear made use of maximum likelihood (ML) (Yang et al., 1995). ML offers a number of advantages over the MP paradigm, both for inferring tree topologies and reconstructing ancestral character states. The first major advantage lies in the fact that ML approaches make use of an explicit model of character state evolution. Because of this, issues with compositional bias and transition to transversion ratios are minimized, as long as a reasonable model of evolution is chosen. ML approaches also take into account branch lengths, estimating them using the chosen model of evolution. Due 
to these factors (assuming adequate model choice), ML has been shown to be a substantial improvement over MP approaches (Yang et al., 1995; Zhang and Nei, 1997; Randall et al., 2016). Further refinements to ML approaches that incorporate data such as rate heterogeneity among sites and joint reconstructions have also been developed and are widely available in popular software packages such as PAML and FastML (Yang, 2007; Ashkenazy et al., 2012).

A third class of reconstruction techniques have also been developed. This class uses Bayesian MCMC techniques to estimate the posterior distribution of substitutions that have occurred over the course of the phylogeny, and were first reported in the early 2000s (Huelsenbeck and Bollback, 2001) . This fully Bayesian approach is very similar to ML methods, with the exception of the fact that it considers uncertainty in all parameters and requires some assumptions about the parameters involved due to the use of a prior. Considering the uncertainty in parameters can be an important improvement, in particular when sample sizes are small. In general, the accuracies of Bayesian and ML approaches are similar for ancestral reconstructions, with both being an improvement on MP approaches (Randall et al., 2016).

An interesting extension to Bayesian ancestral reconstructions is the inference of "substitution mappings". First described by Rasmus Nielsen (2002), substitution mappings assign states not only to nodes within a tree, but also place substitutions along branches. This allows for estimates of the exact timing of substitutions, as well as the possibility of having multiple substitutions on one branch (Figure 1.1). In addition to this, many possible substitution mappings are sampled (one for each draw from the posterior). This helps to accommodate uncertainty in the mappings and minimize potential biases that would be seen when using only the most likely substitution history (Eyre-Walker, 1998; Yang, 2006). Recent implementations of Bayesian substitution mapping approaches in PhyloBayes (Lartillot et al., 2009) and 
PhyloBayes MPI (Lartillot et al., 2013) have made these substitution mappings easily accessible and, with the MPI version, quick to infer.

While reconstructed ancestral sequences have found substantial use in the realm of synthesizing ancient proteins to determine their functions, much less work has been done on computational studies of molecular evolution that make use of the substitution mappings, though there are some examples in the literature. For example, Nielsen and Huelsenbeck used substitution mapping to count the number of non-synonymous and synonymous substitutions at each site in an influenza dataset. By using posterior predictive p-values, they were able to identify sites under positive selection that agreed with previous analyses done using PAML, despite the very different methodologies used (Nielsen and Huelsenbeck, 2002).

Additionally, some work has also been done using substitution mappings to detect coevolving sites. Dutheil et al. created substitution mappings to count how many substitutions occurred on each branch of a tree at different sites, and used the correlations between numbers of substitutions on each branch to infer which sites within RNA were coevolving (Dutheil et al., 2005). Similarly, by creating substitution mappings and using several different test statistics and posterior predictive p-values, Dimmic et al. were able to find coevolving sites in amino acid data (Dimmic et al., 2005). The choice of substitution mappings instead of only ancestral state reconstruction makes sense due to the incorporation of uncertainty handled by sampling many mappings, as well as the fact that multiple substitutions along a branch can be seen, allowing for a more comprehensive view of the substitution process. Despite the success of these studies, molecular evolution work that makes use of substitution mappings has not been a common sight in the literature in recent years, with some exceptions (Jobson et al., 2010; Romiguier et al., 2012; Lemey et al., 2012; Dutheil 


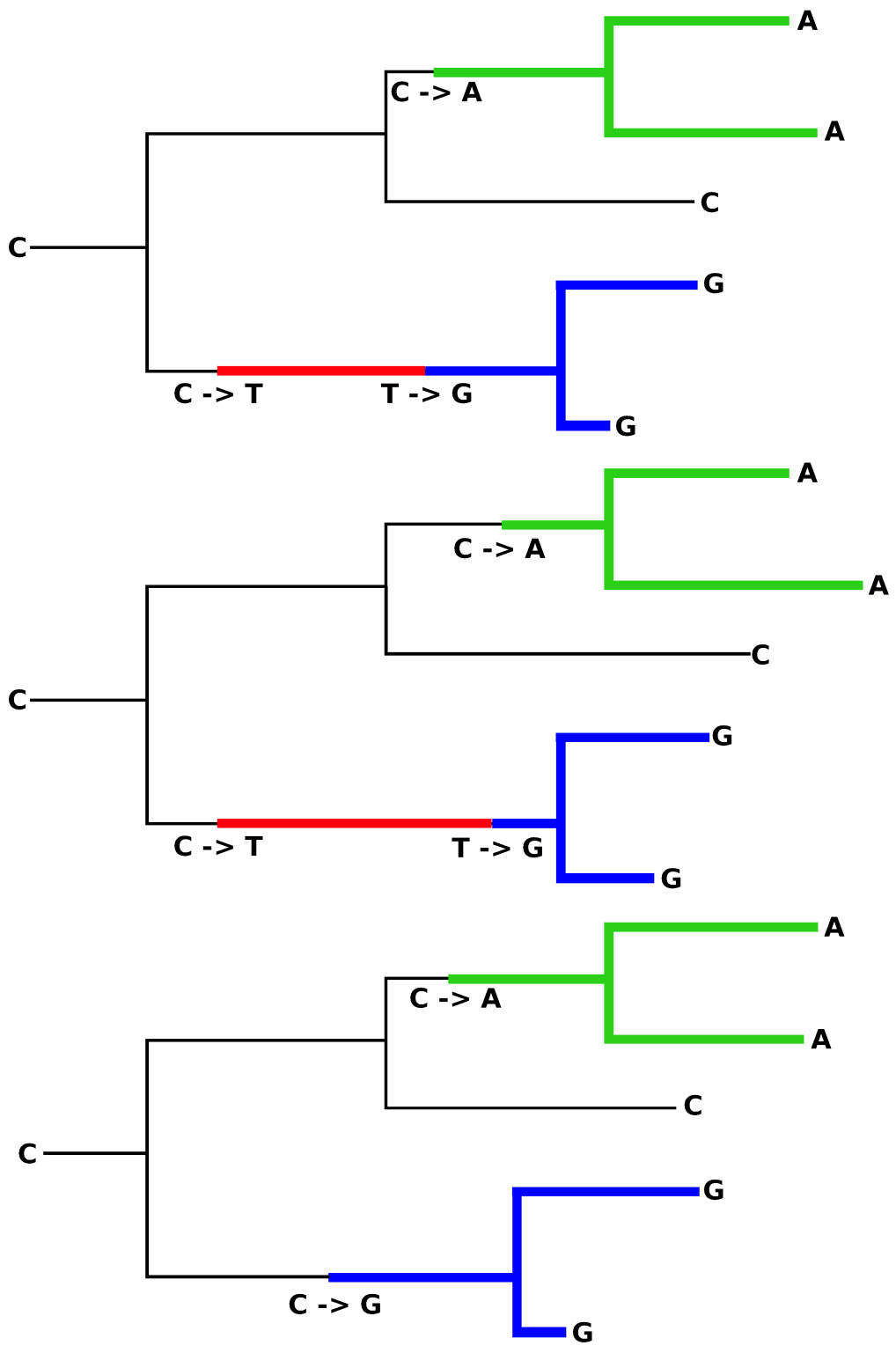

Figure 1.1: An example of a set of substitution mappings for a site, each representing a different draw from the posterior. For the site shown here, the ancestral state was cytosine, and all substitutions for each draw, including multiple changes along a branch, are shown. 
et al., 2012; Romiguier et al., 2013; Lee et al., 2015, 2016).

A number of advantages are gained when using substitution mappings to study molecular evolution. The most obvious advantage to substitution mapping approaches is their speed, when compared to traditional methods. A good example of this speed advantage comes when attempting to calculate omega for a gene of moderate size, in the case that different values of omega should be retrieved for different clades of the tree. Using popular software such as PAML this process is slow, particularly for large datasets, as a different model must be used for each clade of interest, and incorporating this many parameters may over-parameterize the method. Using substitution mappings to solve the same problem is far simpler; a homogenous model can be used to generate the substitution mappings themselves, and the mappings simply read through to count the number of synonymous and non-synonymous changes in each clade. This approach avoids over-parameterization, and is over 60 times faster than classical approaches (Romiguier et al., 2012).

While this speedup is useful, it should be noted that the results should be interpreted with care due to the non-parametric nature of simply reading through substitution histories and counting events. Certain initial states can cause biases which are not accounted for. For example, when characterizing radical versus conservative amino acid substitutions using a PAM or BLOSUM matrix, any site that is a tryptophan will appear to shift very radically any time a substitution is made; while it may be the case that the substitution is radical relative to substitutions at other sites, it may be one of the least radical changes available to the site. One way to mitigate effects such as this is to examine many draws from the posterior instead of only one. This way, many substitution histories are sampled instead of only the most likely history, leading to a more comprehensive view of the substitution history which helps to eliminate biases. For example, perhaps at one node in a tree a tryptophan 
is inferred with relatively weak support, but still more likely than any other amino acid. If only one mapping is examined, this would have the potential to cause major bias. However, when examining multiple mappings this issue is less serious, as many of the mappings will have amino acids other than tryptophan inferred.

As alluded to earlier, Bayesian techniques can use posterior predictive p-values to estimate significance. By simulating data under the same model as that used for the real data, but without the constraint of the real data, the values of the real and predictive distributions can be compared and significance estimates obtained (Nielsen and Huelsenbeck, 2002; Bollback, 2005). When compared to classical model-based methods, this can be construed as an advantage or disadvantage. The advantage of posterior predictive p-values comes from there speed and general use - they can be used whenever it is possible to use posterior predictive simulations. On the other hand, defining test statistics for use with posterior predictive p-values can be difficult, depending on the hypothesis to be tested, and their use can be somewhat contentious (Bollback, 2005). This is a stark contrast to model-based approaches, where methods such as likelihood-ratio tests are available for estimates of significance. The statistical properties of such tests are very well studied and characterized, making them excellent choices for hypothesis testing if one chooses to use a parametric approach.

The studies that make up this thesis investigate the usefulness of Bayesian substitution mappings. Chapter 2 describes the creation of a new sequence simulator, COMPASS, which allows for complete flexibility in both which character states are evolving and the model they evolve according to. COMPASS is used extensively in subsequent chapters. In chapter 3, an attempt is made to find coevolving residues using the mutual information contained within substitution mappings. This approach performs poorly across most relevant biological settings. Finally, chapter 4 investigates differences in the levels of radicality of amino acid substitutions in large versus 
small mammal populations in order to test the nearly neutral theorys prediction that small population sizes should generally have more radical amino acid substitutions. Simulated data using mutation-selection models shows that substitution mappings are able to find differences in radicality with moderate to large differences in effective population size, and in a real dataset of 38 mammals significant differences in radicality are found between large and small population sizes. Substitution mappings are found to be a useful tool that could be applicable for some types of studies in molecular evolution. 


\section{Chapter 2}

\section{COMPASS: The COMPletely Arbitrary Sequence Simulator}

This work has been submitted by Andrew Low, Nicolas Rodrigue, and Alex Wong to Bioinformatics as an Applications Note, and is currently under review.

\subsection{Abstract}

Summary: Simulated sequence alignments are frequently used to test bioinformatics tools, but current sequence simulators are limited to defined state spaces. Here, we present the COMPletely Arbitrary Sequence Simulator (COMPASS), which is able to simulate the evolution of absolutely any discrete state space along a tree, for any form of time-reversible model.

Availability and Implementation: COMPASS is implemented in Python 2.7, and is freely available for all platforms at http://labs.carleton.ca/eme/software-and-data. 


\subsection{Introduction}

Simulated data are often used to assess the performance of new bioinformatic algorithms, test the behavior of biological models, and to generate null distributions through parametric bootstrapping. For example, multiple sequence alignments simulated under known parameter values are used to test the accuracy and robustness of methods for statistical inference from phylogenetic data (Yang and Nielsen, 2000; Lartillot et al., 2009). There are a number of packages available for the simulation of sequence alignments, but they tend to operate over a defined state space and/or implement specific evolutionary models. For example, INDELible offers substantial flexibility with respect to models, and accounts for substitutions, deletions, and insertions, as well as rate heterogeneity among lineages as well as sites. However, its state space is limited to nucleotides, amino acids, and codons; it is unable to, for example, simulate coevolving states (Fletcher and Yang, 2009).

Here, we present COMPASS, a tool for simulating evolution over any arbitrary discrete state space, under any time-reversible evolutionary model along a phylogenetic tree. COMPASS allows for simulation under established rate matrices, and allows for straightforward implementation of novel models of nucleotide, protein, or codon evolution, processes of coevolution between state pairs, or the evolution of phenotypic characters.

\subsection{Methods}

COMPASS is implemented in Python 2.7, and is implemented as command-line program, with all modules used available as part of the source code. It utilizes the Phylo module of BioPython (Talevich et al., 2012) to traverse trees, and a jump 
chain to simulate evolution of characters on a site-by-site basis (Yang, 2006). Userprovided matrices are normalized so that branch lengths are equal to the expected number of substitutions. The rate matrices used by COMPASS can contain absolutely any symbols composed of ASCII characters (i.e. the user is not limited to nucleotides/proteins/codons), and the transition parameters can be any combination of frequencies and other completely arbitrary multipliers.

Standard options such as continuous and discrete gamma distribution of rates, invariant sites, partitioning of data into different models across both sites and branches, and rate heterogeneity among branches are available. Additionally, COMPASS can accept many standard tree formats (newick, nexus, and phyloxml) for input, as well as outputting in a number of standard sequence alignment formats (clustal, fasta, phylip, phylip-sequential, and stockholm, as well as tab-separated). This allows for many rich models of evolution to be implemented, such as mutation-selection models with either constant population size or varying population sizes over different branches of the tree. Full details are given in the user manual, which can be found with at labs.carleton.ca/eme/software-and-data.

\subsection{Verification}

COMPASS has been validated to ensure that the parameters entered will be accurately reflected in the simulated alignments. All parameters are reliably recovered. Data for nucleotide exchangeabilities, equilibrium frequencies, total branch length of input trees, and dN/dS are shown in Figure 2.1. The dN/dS ratio was recovered using the codeml module of PAML 4.8 (Yang, 2007) under an M0 model, while actual values for branch lengths, exchangeabilities, and equilibrium frequencies were recovered by running PhyloBayes MPI (Lartillot et al., 2013) for 1500 generations, taking the 
first 300 iterations as burn-in. Full details can be found in the appendix.

\subsection{Example Usage: Coevolution Simulation}

One area for which COMPASS is well-suited is in creating coevolution data. Identification of coevolving residues can contribute to protein structure prediction, RNA secondary structure prediction, identification of interacting sites between proteins, and protein interaction and protein complex predictions (Shang et al., 2012; Taylor et al., 2012; Weigt et al., 2009). However, there are few tools available to simulate coevolution data. PHASE (Gowri-Shankar and Rattray, 2007) can simulate coevolution for nucleotides, but not for amino acids or codons. MSAvolve (Ackerman et al., 2012) simulates coevolving protein data, but this evolution is not simulated along a tree, rendering it less useful for testing phylogenetically-informed methods. COMPASS addresses all of these gaps.

The first step to using COMPASS is the creation of a parameter file that contains the frequencies of whatever character states one wishes to use, as well as a rate matrix specifying exchangeabilities between character states. These parameter files are simple text files that can be created in any text editor, allowing anybody to create completely new models or any reimplementation of existing models. In the case of nucleotide coevolution, a 16 by 16 rate matrix would be created, while for amino acid and codon coevolution 400 by 400 and 3721 by 3721 matrices, respectively, would be created. Complete descriptions and examples of these files and how to create them can be found at github.com/WongLabPrograms. Once parameter files have been created, COMPASS can simulate with the same model across all sites and branches, different models across sites, different models across branches, or any combination of models across sites and branches. 

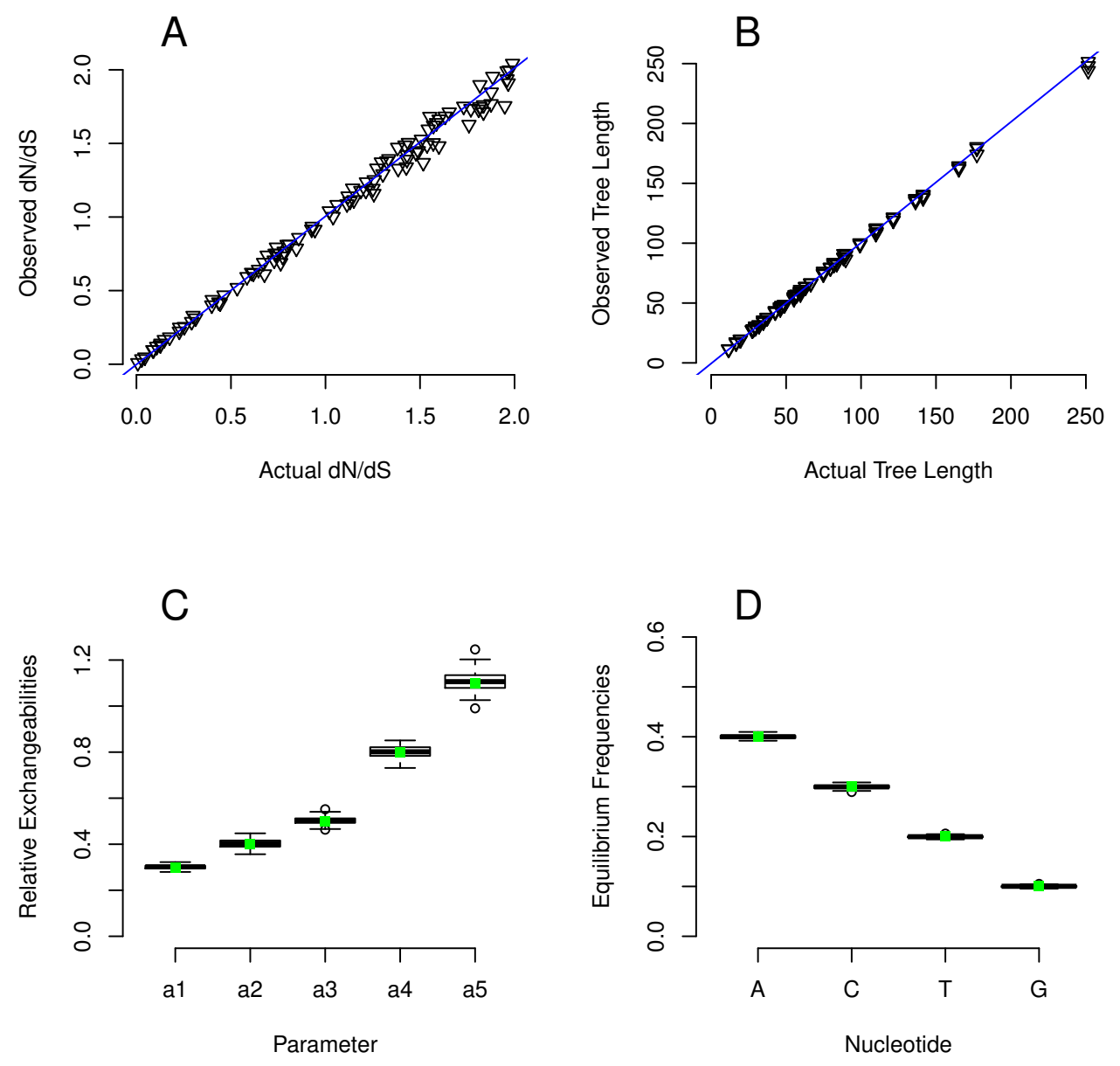

Figure 2.1: 1A) Actual versus observed values of the $\mathrm{dN} / \mathrm{dS}$ ratio. As expected, at lower (more realistic) values, correlation is extremely tight, while the relationship is noisier at higher values of $\mathrm{dN} / \mathrm{dS}$. Tests were carried out with an GY94-style matrix, a 50 species tree, and 150 codons. 1B) Actual versus observed total branch length of trees input into COMPASS. COMPASS very accurately simulates the total tree length. Sequences were evolved under a GTR model with 1000 sites. 1C) Observed relative exchangeabilities for 130 sequences with 1000 sites under a GTR model (the sixth exchangeability was set to 1 and used to benchmark the others). Green squares represent the actual values. 100 replicates were carried out. 1D) Observed equilibrium frequencies for 130 sequences with 1000 sites, evolved under a GTR model. Green squares represent the actual values. 100 replicates were carried out. 
As COMPASS is implemented in Python, it is a tool that the bioinformatics community will easily be able to customize and extend as needed. As COMPASS simulates evolution site-by-site it would, for example, be relatively simple to parallelize it in order to enable fast simulation of very long (i.e. genome length) sequences, and use in Approximate Bayesian Computation (ABC) or Posterior Predictive Simulations (PPS). In addition, functionality for the creation of insertions and deletions could be added, making COMPASS even more usable in general cases as well as situations in which custom rate matrices are required. COMPASS fills a gap in current sequence simulation methods by being fully customizable and easy to use, giving users the power to quickly create and test new models of evolution, as well as simulating coevolution and any other process that can be modelled using discrete characters. 


\section{Chapter 3}

\section{Detection of Coevolving Residues with Phylogenetic Mutual Information}

\subsection{Abstract}

Predicting coevolving residues in nucleic and amino acid data is an open problem in bioinformatics. Here, I develop a new method that uses substitution mappings to infer the amount of time that states are shared between sites throughout evolutionary history. Testing on simulated data reveals that this approach works well in a limited range of circumstances, but not in situations that are biologically relevant. This is found to be due to inaccuracies within the inferred substitution mappings, showing that the approach has promise if higher quality mappings could be inferred.

\subsection{Introduction}

The ability to reliably find coevolving residues in nucleic acid or amino acid data has long been a goal of bioinformatics. Being able to reliably find coevolving residues using computational tools can help with studies of RNA structure prediction, protein structure prediction, protein complex prediction, identification of interacting sites 
between proteins, and intragenic epistasis (Shang et al., 2012; Taylor et al., 2013; Weigt et al., 2009; Kryazhimskiy et al., 2011). Due to the multitude of uses for tools for computational methods for finding coevolution, many algorithms have been developed to attempt to solve this problem.

Many of the algorithms developed to search for coevolving residues are treeignorant, in that they either completely ignore the phylogeny underlying the sequences, or attempt to correct for the influence of phylogeny post-hoc. Perhaps the most widely used metric that falls into this class is Mutual Information (MI - see Equation 3.2 Methods). MI quantifies how much one site in an alignment informs the state of another site within the alignment; when two sites have high MI, they are thought to be likely to be coevolving. MI and its use in coevolution detection has been thoroughly investigated, and found widespread use (Martin et al., 2005; Baker and Porollo, 2016).

One variation on MI, called MIp, attempts to correct for the influence of phylogeny and fast-evolving sites, both of which tend to create false positives. This is done with the following equation. In this equation, $\bar{x}$ represents the average amount of MI between a site and all others (i.e. $M I(a, \bar{x})$ is the average amount of MI between a and all other sites), and $\bar{M} I$ is the average amount of MI between all pairs of sites.

$$
M I p=M I(a, b)-\frac{M I(a, \bar{x}) M I(b, \bar{x})}{\bar{M} I}
$$

The end result of this correction is that sites that have high values of MI with many other sites due to chance have a large correction applied. It has been shown to be a substantial improvement over uncorrected MI, and is often still used as a benchmark when testing new methods (Dunn et al., 2008). Many methods have been developed 
that have improved on MIp and have found use within the bioinformatics community. Notable examples include direct coupling analysis (Weigt et al., 2009), PSICOV (Jones et al., 2012), CCMPRED (Seemayer et al., 2014), and MetaPSICOV (Jones et al., 2015).

All methods mentioned to this point are tree-ignorant, but methods that are treeaware are also available. Incorporating knowledge of the phylogeny seems like a very sensible thing to do; by knowing what the underlying pattern of evolution is, residues that appear to coevolve solely due to their shared ancestry can be detected and correctly excluded. One particularly intriguing set of methodologies that incorporates phylogeny relies on inferring substitution mappings (or simply the states at all ancestral nodes) for each site and inferring coevolution based on these mappings. For example, by inferring ancestral node states and quantifying how quickly substitutions tended to occur one after the other, Kryazhimskiy and colleagues were able to detect putatively positively epistatic residues in influenza proteins (Kryazhimskiy et al., 2011). Similarly, by inferring substitution mappings and then finding correlations between the number of substitutions detected using these mappings between sites, contacts in RNA were reliably predicted (Dutheil et al., 2005). This method was later extended to be able to work with amino acid data and find clusters of coevolving residues (Dutheil and Galtier, 2007).

In the same vein as this work, Dimmic et al. (2005) generated Bayesian substitution mappings in order to find coevolving sites. To do this, they tested several different metrics, both parametric and non-parametric. Parametric statistics made use of an explicit Markov model of coevolving residues based on an interaction matrix in which substitutions that resulted in more favorable pairings had accelerated rates. Due to the use of this model, they were able to use a likelihood ratio test, which ended up being the most successful metric tested on both simulated data and a real 
dataset of a phosphoglycerate kinase gene. Other metrics used included calculation of the amount of time spent in favourable versus non-favourable states, and mutual information found in the alignment. Notably, this study also made use of posterior predictive p-values in order to estimate significance, an important advantage of Bayesian techniques (Nielsen and Huelsenbeck, 2002; Bollback, 2005).

In this study, we also make use of Bayesian substitution mappings to attempt to find coevolving residues. MI is used as a metric, but instead of being based on the frequencies of residues found in the alignment, it is calculated based on the proportion of time throughout evolutionary history that states are seen together. This metric, termed phylogenetic MI, performs well in simple cases, but performs poorly when complicating factors such as rate variation across sites are put into simulations. Simulated amino acid data is also tested, but performs poorly in all cases.

\subsection{Methods}

\subsubsection{Inferring Substitution Mappings}

To infer substitution mappings, PhyloBayes MPI (Lartillot et al., 2013) was run for at least 2000 generations under a fixed tree topology using a CAT-GTR model with 4 discrete gamma categories. 96 evenly spaced points were taken from the posterior to infer possible substitution mappings, after discarding the first 10 percent of the chain as burn-in.

\subsubsection{Reading Mappings and Calculating MI}

I wrote a Python parser making use of BioPython's Phylo module (Talevich et al., 2012) to read through the substitution mappings and find the mutual information 


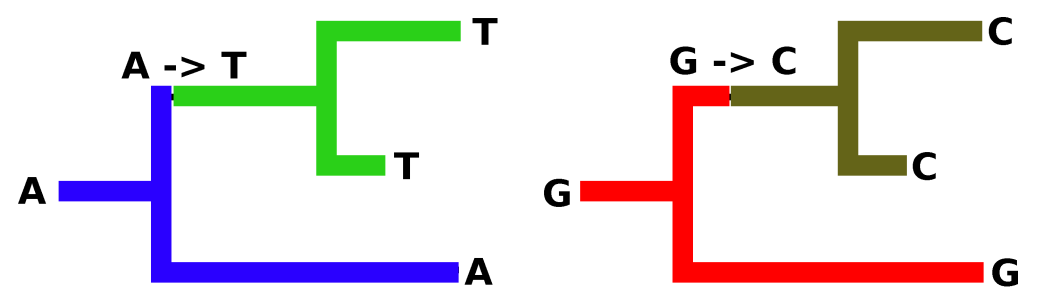

Figure 3.1: A substitution mapping for two different sites. The site on the left is in state A for 46.1 percent of its evolutionary history and state $\mathrm{T}$ for 53.9 percent of its history. The site on the right is 54.5 percent $\mathrm{G}$ and 45.5 percent $\mathrm{C}$. The joint probabilities for $\mathrm{AG}, \mathrm{TG}$, and $\mathrm{TC}$ are 46.1, 8.1, and 45.5, respectively.

(MI) based on the amount of time throughout evolutionary history that states are shared between sites. MI was calculated using the following equation:

$$
M I(a, b)=\sum_{a} \sum_{b} p(a, b) \ln \frac{p(a, b)}{p(a) p(b)}
$$

As an example, consider Figure 3.1. This figure shows two substitution mappings, each for a separate site. By reading along the trees, it can be found that the site on the left is in state A for 46.1 percent of its evolutionary history and state $\mathrm{T}$ for 53.9 percent of its history. The site on the right is 54.5 percent $\mathrm{G}$ and 45.5 percent $\mathrm{C}$, and the joint probabilities for AG, TG, and TC are 46.1, 8.1, and 45.5, respectively. Taking these numbers and plugging them into the MI equation gives us 0.457 , a relatively high value which indicates that the sites may be coevolving. The same equation was used for calculation of alignment MI, but with values based only on those states observed in the sequence alignment.

\subsubsection{Posterior Predictive P-Values}

Posterior predictive p-values were calculated as the number of draws from the posterior for which the null distribution contained more MI than the actual distribution, divided by the total number of draws. The mappings in the null distribution are 
generated along the same tree structure and with the same model as the real data, but without the constraint of the observed data. For all simulations mentioned here, $\mathrm{p}$-values were considered significant at the significance level $\mathrm{p}=0$.

\subsubsection{Data Simulation}

COMPASS (Chapter 2) was used to generate all simulated data. For these simulations, the strength of coevolution was defined as the proportion of time throughout evolutionary history that coevolving sites were set to be in a preferred paired state. All simulations involved 40 sites evolved independently and 20 coevolving pairs, for a total of 80 sites. When rate variation across sites was incorporated, 4 discrete gamma categories were used.

\subsubsection{Metrics Used For Algorithm Evaluation}

To evaluate performance of the algorithms used here, the metric of choice was Positive Predictive Value (PPV). PPV (also known as precision) is the proportion of pairs predicted to be coevolving ( $\mathrm{p}$-value $=0)$ that are truly coevolving, and is calculated with the following:

$$
\text { PPV }=\frac{\text { Number of True Positives }}{\text { Number of True Positives }+ \text { Number of FalsePositives }}
$$

PPV is used due to the fact that coevolution detection presents a large class imbalance; there are far more pairs that are not coevolving than pairs that are coevolving. This means that using metrics such as sensitivity (the proportion of coevolving sites that are successfully detected) and specificity (the proportion of independently evolving sites that are successfully excluded) can be misleading, as even with high values 
for both sensitivity and specificity many false positives can be predicted.

\subsection{Results and Discussion}

To begin, algorithm performance was evaluated on nucleotide data with no rate variation across sites, the simplest possible case. Tests were carried out at many different levels of coevolution strength. When simulated on a fixed 130-species tree generated with a birth-death process using PHASE (Gowri-Shankar and Rattray, 2007). At an average branch length of 0.34 , values of PPV were strong at all levels of coevolution strength tested (Figure 3.2) Notably, sensitivity for phylogenetic MI fell to very low levels (0.05) at low coevolution strengh, while sensitivity for alignment MI was a minimum of 0.5 at all strengths tested. Full details on the rate matrices used can be found in Appendix A.

As rate variation across sites is a common phenomenon, the effect of rate variation on algorithm performance was assessed. Data was simulated along the same 130 -species tree previously used at a coevolution strength of 0.75 , a level at which algorithm performance was excellent when no rate variation across sites was present. Rate variation across sites was simulated with 4 discrete gamma categories and varying values of alpha. At low values of alpha (high variance in rates), performance was poor, with performance increasing with increasing rate homogeneity, and phylogenetic MI was consistently outperformed by alignment MI with the MIp correction applied (Figure 3.3).

The effect of branch lengths within a tree was also assessed. Beginning with the same tree used in previous sections, homogenous rates across sites and a coevolution strength of 0.75 , branches were both contracted and elongated. Both contraction and 


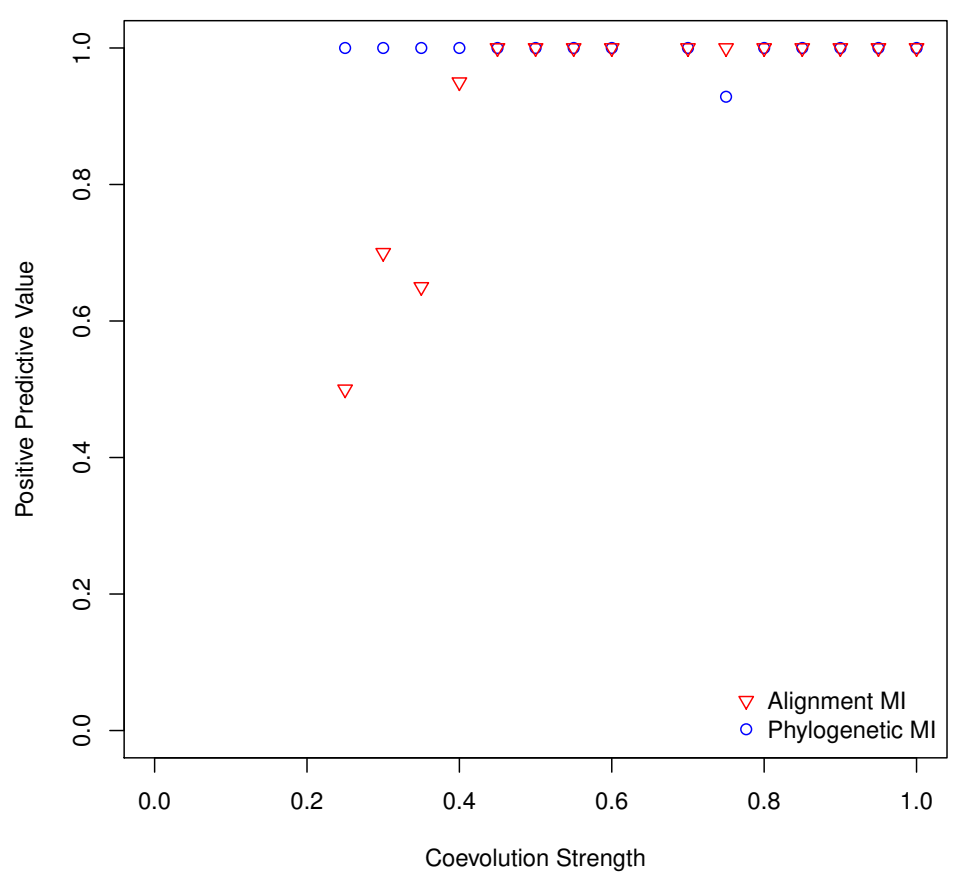

Figure 3.2: PPV versus coevolution strength for a 130 -species tree with no rate variation across sites. PPV is strong at all levels of coevolution strength tested. 


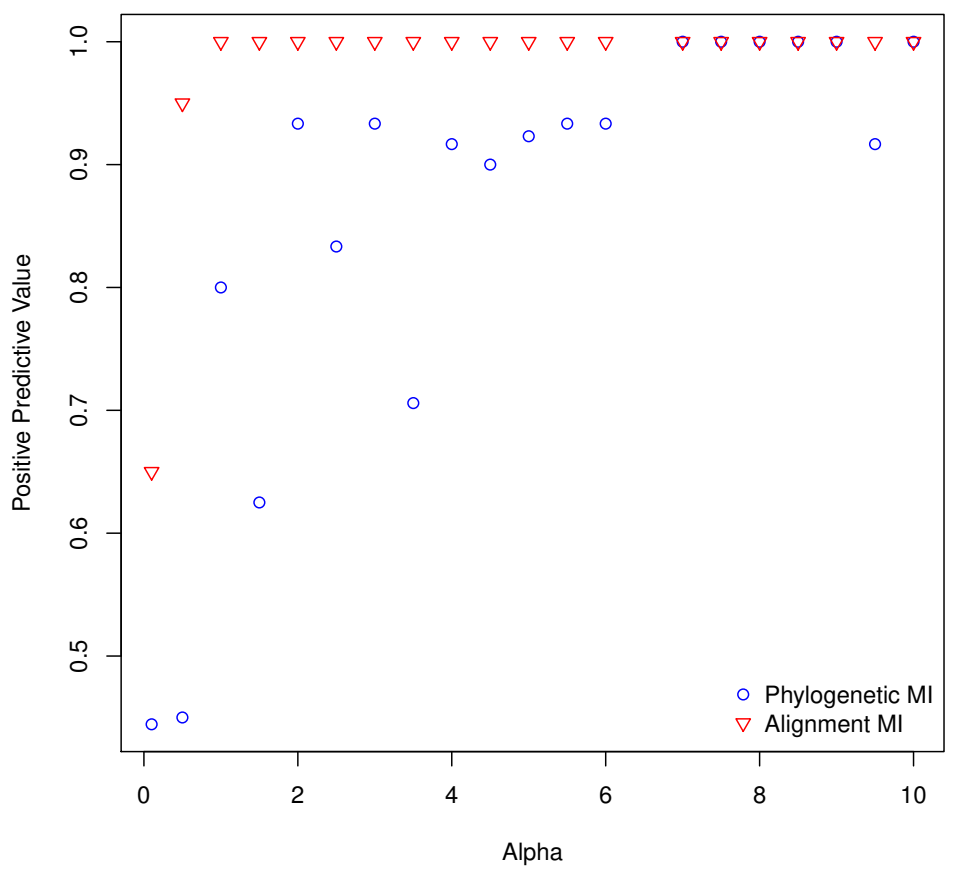

Figure 3.3: Effect of varying values of alpha on PPV for a 130 -species tree. When rate variation across sites is high (low alpha values), PPV is poor. As rates across sites become more homogenous, performance sees substantial improvement, but still tends to underperform when compared to alignment MI with the MIp correction applied. 


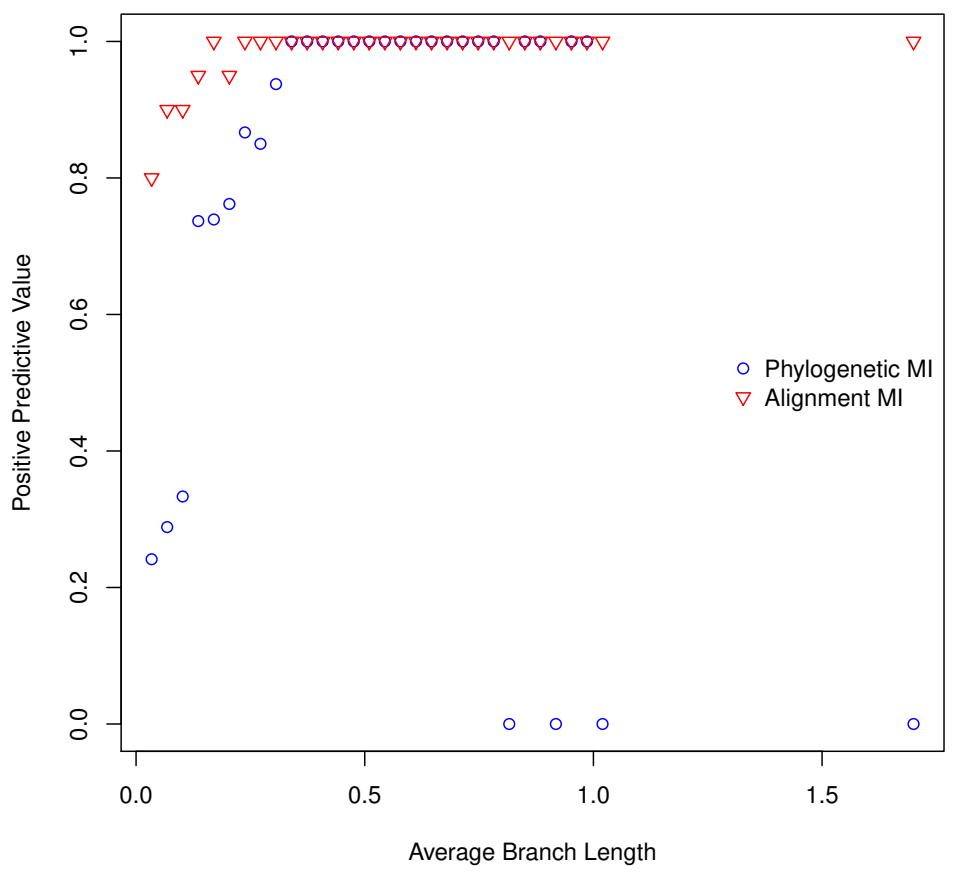

Figure 3.4: Effect of contracting and elongating branches on PPV. Beginning with a 130 -species tree with average branch lengths of 0.34 , branches were contracted to an average as short as 0.034 and elongated to up an average as long as 1.7. PPV values were low with both very short and very long branches, with optimal performance at average branch lengths between 0.3 and 0.7 . At all branch lengths, phylogenetic MI performed worse than alignment MI with the MIp correction.

elongation of branches lowered PPV substantially, with the algorithm performing best when average branch lengths were between 0.3 and 0.7 , and losing all power when average branch length approached 1.0. As with investigation into rates across sites, phylogenetic MI was outperformed by alignment MI with the MIp correction applied. (Figure 3.4).

In a final test on simulated nucleotide data, a variety of alpha parameters were tested on the branch lengths previously found to be optimal (0.3 to 0.7) at a coevolution strength of 0.75 . Similar to previous testing, performance was generally 


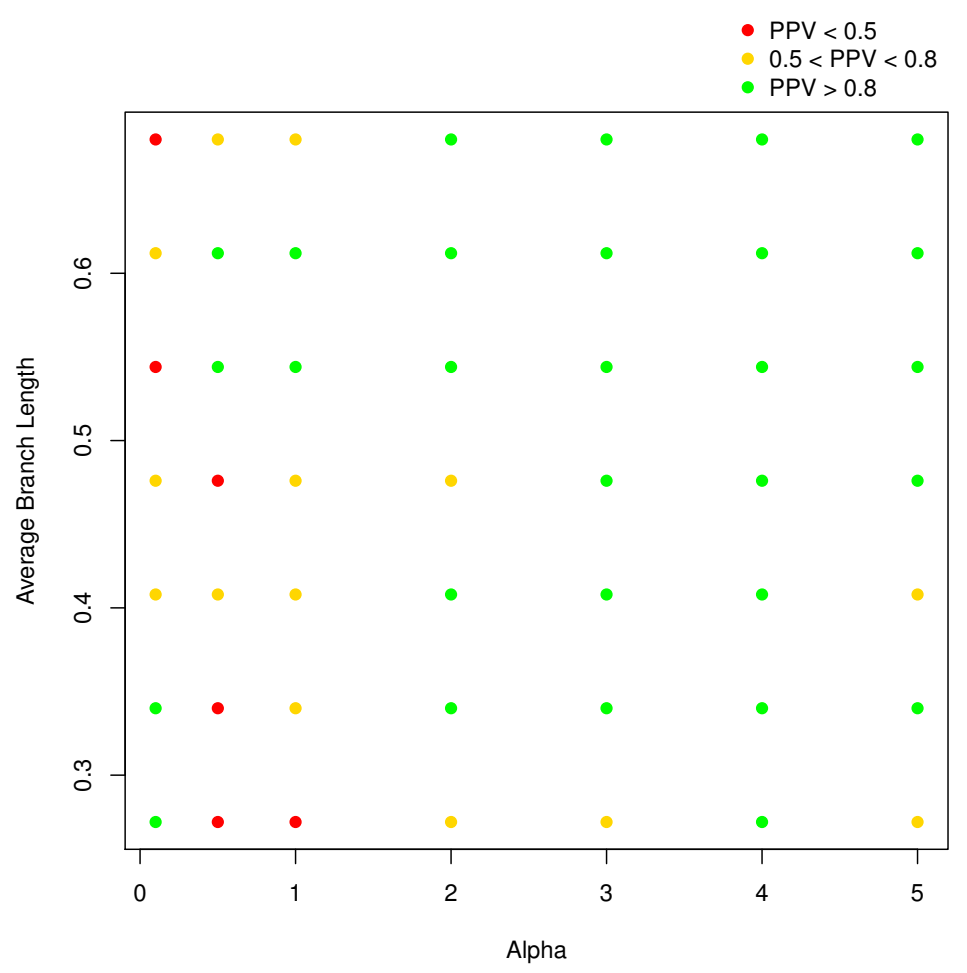

Figure 3.5: Effect of alpha values on PPV within the optimal branch length range. As expected, performance tends to be poor when rate variation across sites is high, and improves as rates become more homogenous.

poor when rate variation across sites was high, with improvement as rates across sites became more homogenous (Figure 3.5).

Testing was also carried out on simulated amino acid data. In order to gain power, amino acid data required trees with more branches than nucleotide data, even with no variation in rates across sites and branch lengths previously found to be optimal. Phylogenetic MI was outperformed by alignment-based MI with the MIp correction at every level of coevolution strength when tested on a 500-species tree (Figure 3.6). Full information on the rate matrices used can be found in Appendix A.

The idea of calculating MI based on phylogenetic instead of sequence information has obvious potential advantages. By incorporating knowledge of the phylogeny, the 


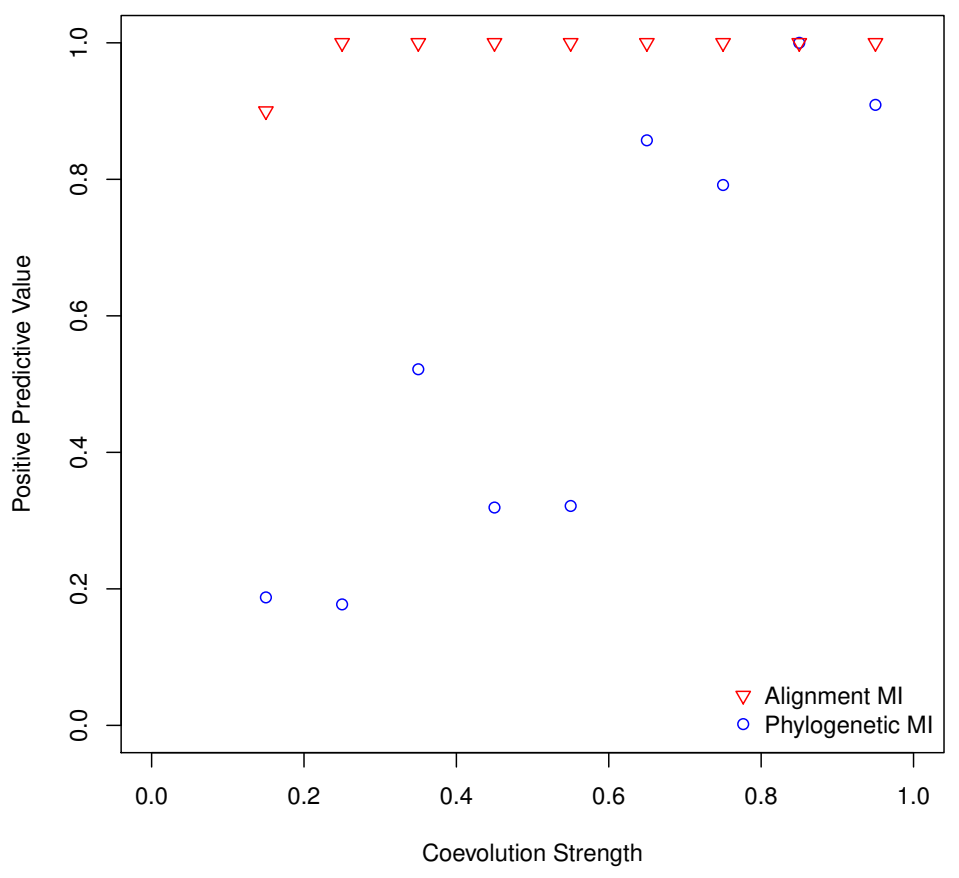

Figure 3.6: A comparison of PPV for alignment-based MI with the MIp correction applied and phylogenetic MI on a 500-species tree with no rate variation across sites. Alignment-based MI outperforms phylogenetic MI at every level of coevolution strength tested. 
underlying evolutionary relationships are accounted for; this should help to reduce the number of false positives that arise solely due to shared ancestry, a major issue with alignment-based MI for which corrections have had to be developed (Dunn et al., 2008). Additionally, posterior predictive p-values are able to provide estimates of confidence for each potentially coevolving pair when using phylogenetic MI, whereas alignment-based MI relies only on the magnitude of the MI found. Due to this, an arbitrary cut-off must be set to determine which residues are coevolving and which are not.

Despite these advantages, phylogenetic MI performs poorly in almost all situations tested here. The poor performance of phylogenetic MI may be due to inaccurate reconstruction of the actual substitution history. To test this hypothesis, the true mappings generated by COMPASS were compared to the mappings inferred by PhyloBayes. A 500-species amino acid alignment at coevolution strength 0.45 was created, with rate variation across sites using 4 discrete gamma categories at an alpha value of 1 . This is a situation for which performance is very poor when using inferred mappings. When the true mappings created by COMPASS are compared to the inferred mappings, it can be seen that PhyloBayes consistently underestimates MI for coevolving pairs (Figure 3.7). This verifies the fact that if mappings were able to be inferred with complete accuracy, phylogenetic MI would be a far more powerful method than it is in its current state.

This brings about the question of why the mappings produced by PhyloBayes are not of sufficient quality to correctly identify coevolving residues. One obvious issue is that the underlying model used to infer the substitution mappings does not account for dependencies between residues; each site is assumed to be independent. Development of models that account for dependencies between sites could help to 


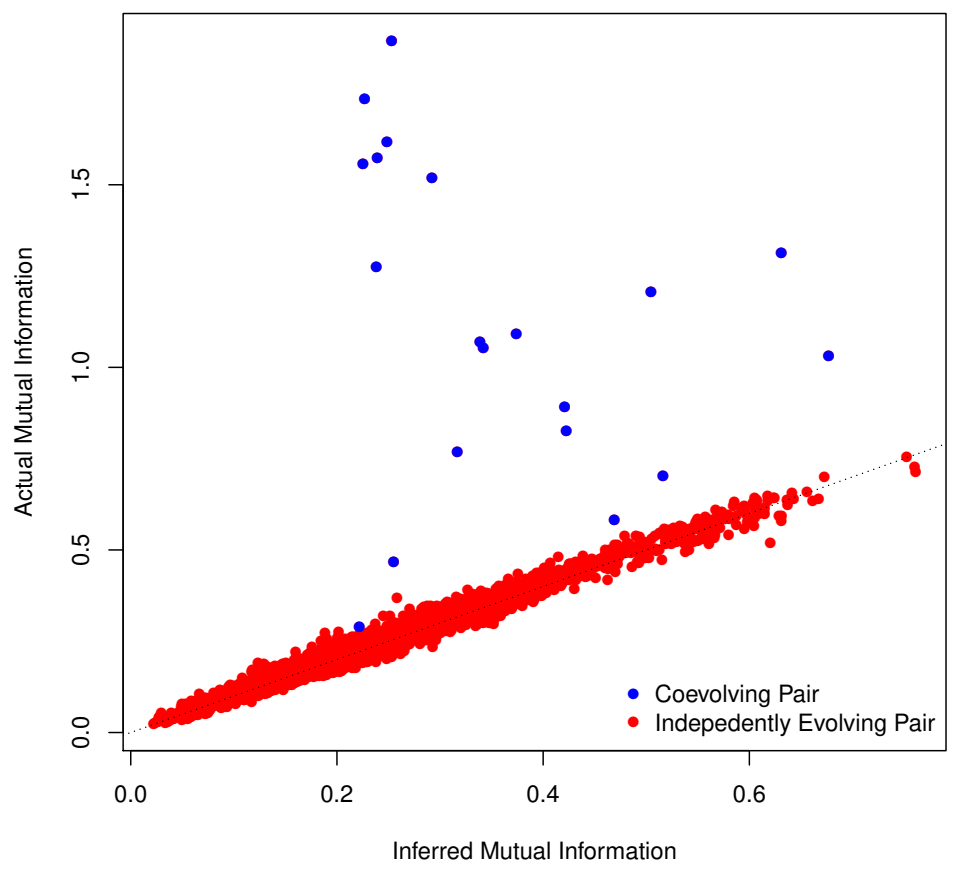

Figure 3.7: Actual MI plotted against the MI inferred by PhyloBayes. Correlation among independent sites (in red) is very tight, while the amount of MI present between coevolving sites (shown in blue) is consistently underestimated. The dashed line shows a 1:1 correlation. 
alleviate this problem, and some models have been proposed that are able to do this for nucleic acid sequence data (Dib et al., 2014). Models such as this could be extended to amino acid or codon data, although this would be difficult due to the computational complexity of the problem arising from the size of the matrices that would need to be used (400 by 400 for amino acids, 3721 by 3721 for codons).

Exacerbating any issues with the mappings is the fact that using phylogenetic MI requires mappings that are accurate in terms of not only which substitutions are occurring, but also exactly when they occur along branches. This presents an additional challenge when compared to methods that only use states at ancestral nodes (i.e. Kryazhimskiy et al. 2011), which can be inferred with very high accuracy (Randall et al., 2016). Methods that use numbers of substitutions along branches (i.e. Dutheil et al. 2005), also require less precision when inferring the timing of mappings, making approaches that use similar ideas an intriguing area for future development.

Overall, the use of phylogenetic MI for coevolution detection has potential for future use, if mappings that are sufficiently accurate can be developed. More broadly, approaches that make use of substitution mappings are also a promising avenue for research, particularly if such approaches have less reliance on perfect placement of substitutions along branches than phylogenetic MI. 


\section{Chapter 4}

\section{Substitution Mapping Shows Elevated Levels of Radical Amino Acid Substitutions in Large-Bodied Mammals}

This work is in preparation by Andrew Low, Nicolas Rodrigue, and Alex Wong for submission to Molecular Biology and Evolution.

\subsection{Abstract}

Substitution processes that determine the fate of mutations vary widely between genes, genomes and species. Effective population size $\left(\mathrm{N}_{\mathrm{e}}\right)$ is one important factor in this process, with nearly neutral theory predicting that more slightly deleterious mutations will escape purifying selection in small population sizes than in large population sizes. To test this hypothesis, we use previously unused genomic resources and a novel computational method based on substitution mapping in order to quantify how radical amino acid substitutions are between species with large and small population sizes. We find that small population size species are more likely to undergo more radical (and therefore more deleterious) substitutions, as well as having 
elevated levels of non-synonymous to synonymous substitutions, showing support for the nearly neutral theory.

\subsection{Introduction}

Understanding the process of substitution remains a fundamental question in molecular evolution, as rates and modes of evolution can be very different in different genes, genomes, and species (Kimura, 1968; Ohta, 1973; Pollard et al., 2006; Lanfear et al., 2010). Histones, for example, are extremely conserved between even distantly related species (DeLange et al., 1969), while genes involved in immune and reproductive function are often highly diverged in closely related species (Hughes and Nei, 1988; Swanson and Vacquier, 2002).

One important parameter in the substitution process is effective population size $\left(\mathrm{N}_{\mathrm{e}}\right)$. In the context of codon evolution, Equation 4.1 shows how the rate matrix $Q_{i j}$ can be modelled as a product of $\mathrm{N}_{\mathrm{e}}$, exchangeabilities between nucleotides $(\epsilon)$, codon equilibrium frequencies $(\pi)$, and probabilities of fixation (Yang and Nielsen, 2008).

$$
Q_{i j}=2 N_{e} * \epsilon_{i j} * \pi_{j} * p(f i x)
$$

Fixation probability can be approximated with Equation 4.2, where $s_{i j}$ is equal to the difference in fitness between codon $i$ and codon $j$ (Fisher, 1930; Wright, 1931).

$$
p(f i x)=\frac{s_{i j}}{1-e^{-2 N_{e} s_{i j}}}
$$

Substituting Equation 4.2 into Equation 4.1 yields Equation 4.3.

$$
Q_{i j}=\epsilon_{i j} \pi_{j} \frac{2 N_{e} s_{i j}}{1-e^{-2 N_{e} s_{i j}}}
$$


In Equation 4.3 the role that $\mathrm{N}_{\mathrm{e}}$ plays in the substitution process becomes clear; when population sizes are small, the last term in the equation will be very close to one unless selection coefficients are very large. This shows clearly that selection is weak when $\mathrm{N}_{\mathrm{e}}$ is small, as selection becomes almost entirely independent of population size. Conversely, as $\mathrm{N}_{\mathrm{e}}$ grows to large numbers the final term in the equation becomes large even when selection coefficients are small. This shows that with large population sizes selection becomes the dominant force, even when selection coefficients are small.

This result can be applied to test the nearly neutral theory, which states that selection coefficients tend to be either strongly deleterious or close to neutral, with only small positive or negative effects (Ohta, 1972b). As the nearly neutral theory posits that many mutations are slightly deleterious, we can expect that in species with small population sizes these mutations will escape selection. On the other hand, in large populations these mutations will be seen by purifying selection, as the large values of $\mathrm{N}_{\mathrm{e}}$ will effectively screen out mutations with even very slight deleterious effects. This argument has been invoked to explain why many proteins evolve in a clock-like fashion; when population size is large there is more mutational supply, but also more efficient selection, leading to similar rates of substitution in small and large population sizes (Ohta, 1972a).

Comparative methods offer a promising approach for testing the prediction that purifying selection acts more efficiently on larger populations, as would be expected under the nearly neutral theory. A few key parameters should have elevated values when purifying selection is weak. In particular, the ratio of non-synonymous to synonymous substitutions $(\mathrm{dN} / \mathrm{dS})$ is expected to be higher when purifying selection is weak, as most non-synonymous substitutions have been shown to be deleterious (Hietpas et al., 2011). Similarly, Kr/Kc, the ratio of radical amino acid changes (typically defined as a substitution that causes a change in the polarity, volume, or 
charge of an amino acid) to conservative amino acid changes (which do not change the polarity, volume, or charge of an amino acid), should be higher in smaller populations where purifying selection is weaker as radical changes tend to be more deleterious than conservative changes (Smith, 2003).

To test the prediction that $\mathrm{Kr} / \mathrm{Kc}$ and Dn/Ds are higher in small population sizes, body mass is often used as a proxy for effective population size, as the two have a strong negative correlation. The expected negative correlation between $\mathrm{dN} / \mathrm{dS}$ and body mass has been found for mammalian mitochondrial genes (Popadin et al., 2007; Lartillot and Poujol, 2011) and nuclear genes (Lartillot, 2013), and for $\mathrm{Kr} / \mathrm{Kc}$ in birds (Weber et al., 2014; Nabholz et al., 2013). Additionally, the dN/dS ratio of plants that were restricted to islands (thereby causing low effective population sizes) was found to be higher than plants found on the mainland (Woolfit and Bromham, 2005). In comparisons between the $\mathrm{dN} / \mathrm{dS}$ ratio of primates and rodents, primates were found to have higher $\mathrm{dN} / \mathrm{dS}$ ratios than rodent species, with the conclusion again being that this disparity was due to differing strengths of purifying selection caused by population size differences (Hughes and Friedman, 2009).

Here, we take advantage of new genomic resources and a novel computational approach to further address the relationship between $\mathrm{N}_{\mathrm{e}}$ and rates of molecular evolution in mammals. We make use of the 100-way alignment of vertebrate genomes available from the UCSC genome browser to investigate correlations between population size and $\mathrm{Kr} / \mathrm{Kc}$ or $\mathrm{dN} / \mathrm{dS}$ across 780 genes in 38 mammalian species. Using a new substitution mapping approach, we find that the average amino acid substitution in large-bodied species (those with small $\mathrm{N}_{\mathrm{e}}$ ) is significantly more radical than the average substitution in small-bodied $\left(\right.$ large $\mathrm{N}_{\mathrm{e}}$ ) species. This evidence is corroborated by an analysis of which finds that small-bodied species have lower value of $\omega$ than large-bodied species, and a strong correlation between $\mathrm{Kr} / \mathrm{Kc}$ and body size. 


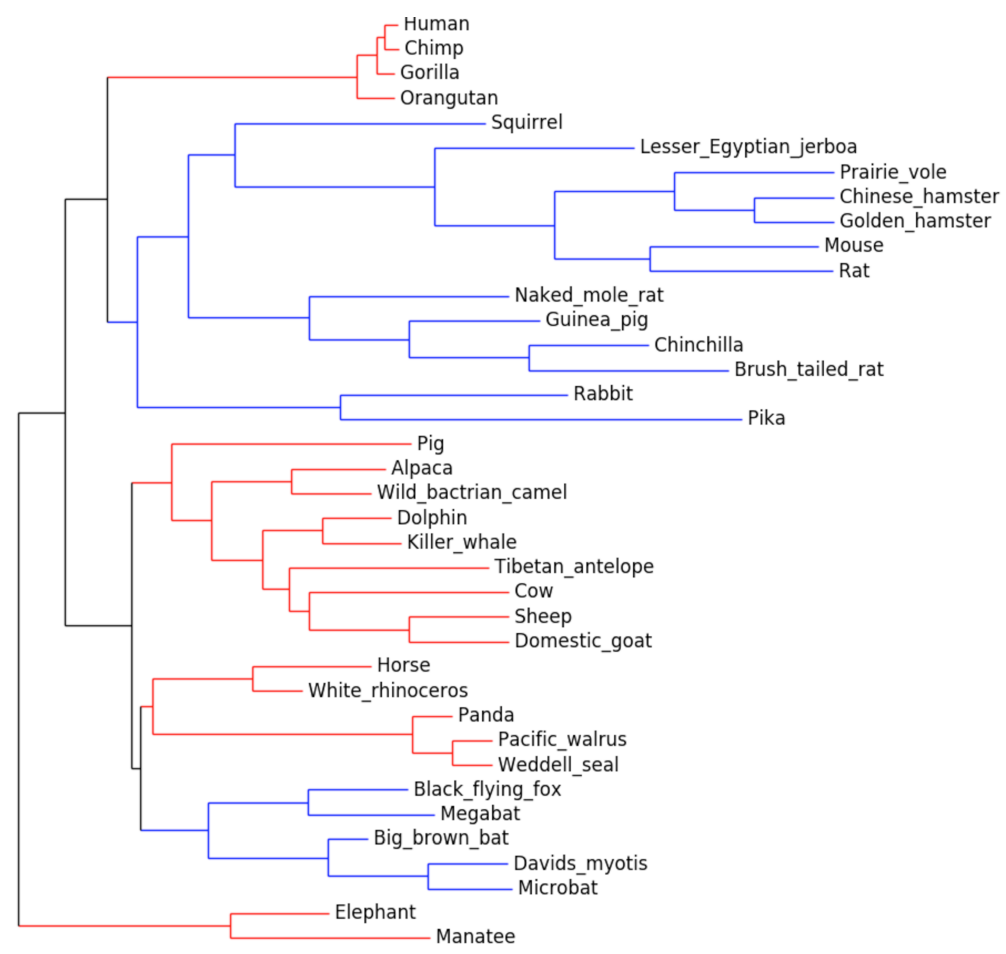

Figure 4.1: The phylogeny used in the present study. Species considered large are found on red branches, while small-bodied species are in blue.

\subsection{Methods}

\subsubsection{Dataset}

Amino acid and nucleotide sequences, as well as the phylogenetic tree used (Figure 4.1), were downloaded from the UCSC Genome Browser's "100 way" 100 verterbrate species alignment (Blanchette, 2004). Sequences which had fewer than 5 percent gaps in all sequences used in in this study were retained, resulting in a dataset of 780 genes (412522 amino acids). Body sizes of mammals within the study were obtained from the AnAge database (Tacutu et al., 2013) 


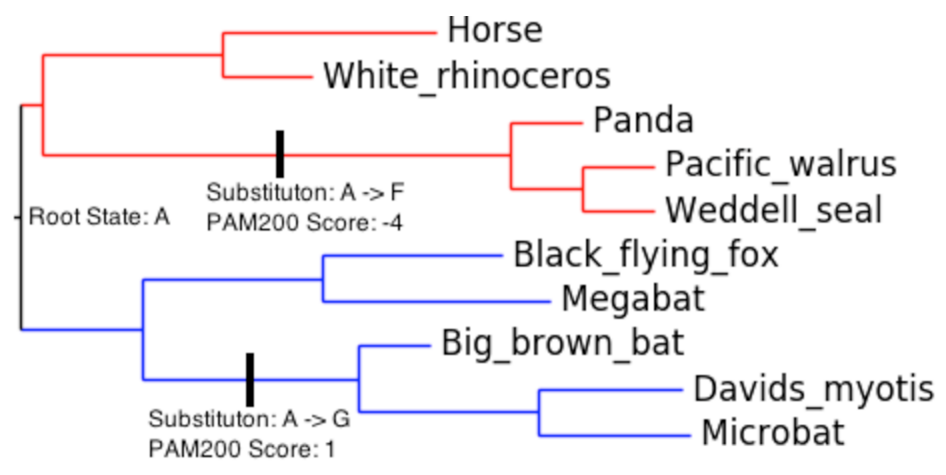

Figure 4.2: An example of a substitution history created by PhyloBayes. At the root of the clade, the site being examined is an alanine. Within the bat clade, an alanine to glycine substitution occurs, and receives a score of 1 , while an alanine to phenylalanine substitution occurs on the branch leading to the panda, walrus and seal, and receives a score of -4 .

\subsubsection{Substitution Mappings}

To quantify the level of radicality of amino acid substitutions, substitution mappings for each gene were created using PhyloBayes MPI (Lartillot et al., 2013). Given a sequence alignment and a tree topology, PhyloBayes will infer all substitution events that occurred from the root of the tree to the tips on a site-by-site basis, allowing for a complete substitution history of each site. It is important to note that this is done for several draws from the posterior, therefore accounting for most (if not all) of the likely substitution maps. Chains were run for 50 generations using a fixed tree topology under a GTR-CAT model with 4 discrete gamma categories, with the first 10 generations discarded as burn-in. Each chain was run twice, and yielded very similar results each time. Scoring of radicality was performed using either PAM or BLOSUM matrices, with each substitution event observed being assigned the corresponding score from the chosen scoring matrix, resulting in radical changes receiving lower scores than conservative changes (Figure 4.2).

The total score for each gene was then divided by the number of events observed 
within the substitution mapping, providing the average level of radicality for each substitution. In order to assess significance, 1000 bootstrap replicates were performed to compare the difference in average radicality between small- and large-bodied species with the difference between randomly selected species. Each bootstrap replicate partitioned species at random into two groups, instead of dividing into two groups based on body size. The tree used, with large-bodied species marked in red and small-bodied species marked in blue, can be found in Figure 4.1.

\subsubsection{Simulation of Data Under Mutation-Selection Models}

To validate our substitution mapping-based approach, simulated data were generated under a mutation-selection model as described in Yang and Nielsen (2008). Nucleotide frequencies and exchangeabilities were obtained from an alignment of mammalian beta-globin sequences found in Yang and Nielsen (2000). Fitness profiles for each codon were taken from one of the 10 profiles found in the $\mathrm{C} 10$ model in PhyloBayes MPI (Lartillot et al., 2013), and simulated data was created using COMPASS (Chapter 2). 100 alignments 200 codons in length were created following the same tree topology as that used in this study, with populations simulated at 20, 100, and 500-fold differences in size, as well as at equal population sizes. The species chosen to have large and small population sizes in our simulated data were the same species determined to have large and small population sizes in our real dataset. Testing of levels of radicality was carried out using the methodology described in the previous section. 


\subsubsection{Correlations Between Body Size and $\mathrm{Kr} / \mathrm{Kc}$}

CoEvol 1.4b (Lartillot and Poujol, 2011), a Bayesian MCMC sampler which aims to find correlations between rates of molecular evolution and observable traits using phylogenetically informed methods, was used to examine the correlation between body size and the radicality of amino acid substitutions. The radicality of substitutions was examined under 4 different models polarity, volume, charge, and a combination of polarity and volume. In all models, a substitution that causes a shift in the physicochemical property being examined is considered to be radical, while substitutions that did not change the property in question are considered to be conservative. Chains were run for 100 generations for each gene for each model, taking the first 10 generations as burn-in, with chains of 1000 generations being run for the polarity-volume model to ensure that shorter chains were providing adequate qualitative results.

\subsubsection{Effects of Body Size on Rates of Nonsynonymous Sub- stitution}

We tested for an effect of body size on $\omega$, the rate of nonsynonymous substitutions relative to synonymous substitutions, using branch models in codeml, part of the PAML package (Yang, 2007). Two different branch models were implemented. In the first (null) model, large and small-bodied clades were constrained to have the same value of $\omega$, while in the second model $\omega$ was permitted to vary between large and small-bodied clades. In both models, branches that did not belong to either small or large-bodied clades had a separate $\omega$. To assess the fit of the second model over the null, a log-likelihood ratio test was performed. 


\subsection{Results}

We propose to investigate changes in substitution radicality using a mapping approach, whereby a posterior distribution of substitution histories is used to make inferences concerning the radicality of amino acid changes. We validated this strategy using simulated data, with simulations allowing for a range of differences in $\mathrm{N}_{\mathrm{e}}$. As can be seen in Figure 4.3, when population sizes are equal or have a 20-fold difference in size (Fig. 4.3A and 4.3B) the distribution of radicality scores shows essentially no difference between large- and small- $\mathrm{N}_{\mathrm{e}}$ species, and is not significantly different (Kolmogorov-Smirnov, $\mathrm{p}=0.9139$ for equal populations, $\mathrm{p}=0.4449$ for 20 fold difference). When the difference in population size grows to 100-fold (Fig. 4.3C), large-bodied species tend to have more radical substitutions than small-bodied species (Kolmogorov-Smirnov, $\mathrm{p}=0.0006$ ), and at a 500-fold difference (Fig. 4.3D) in population size the separation in radicality between large and small species becomes very clear (Kolmogorov-Smirnov, $\mathrm{p}=2.2 \mathrm{e}-16$ ). Thus, our approach has the power to detect differences in substitution history owing to modest and large changes in $\mathrm{N}_{\mathrm{e}}$.

We then applied the substitution mapping approach to compare the radicality of amino acid changes in mammalian species with large bodies (and hence small population sizes) versus those with small bodies (and hence large population sizes). Large-bodied species tended to have more radical substitutions than small-bodied species (Figure 4.4). The average score for small-bodied species was 1.10 using a PAM 200 matrix, while in large-bodied species the average score was 1.03, indicating that the average substitution in large-bodied species involved a more radical amino acid shift than the average substitution in small-bodied species, a significant difference (Kolmogorov-Smirnov, $\mathrm{p}=0.0289$ ). The average difference of 0.07 between small and large-bodied species in this dataset was similar to the difference of 0.08 observed in our 

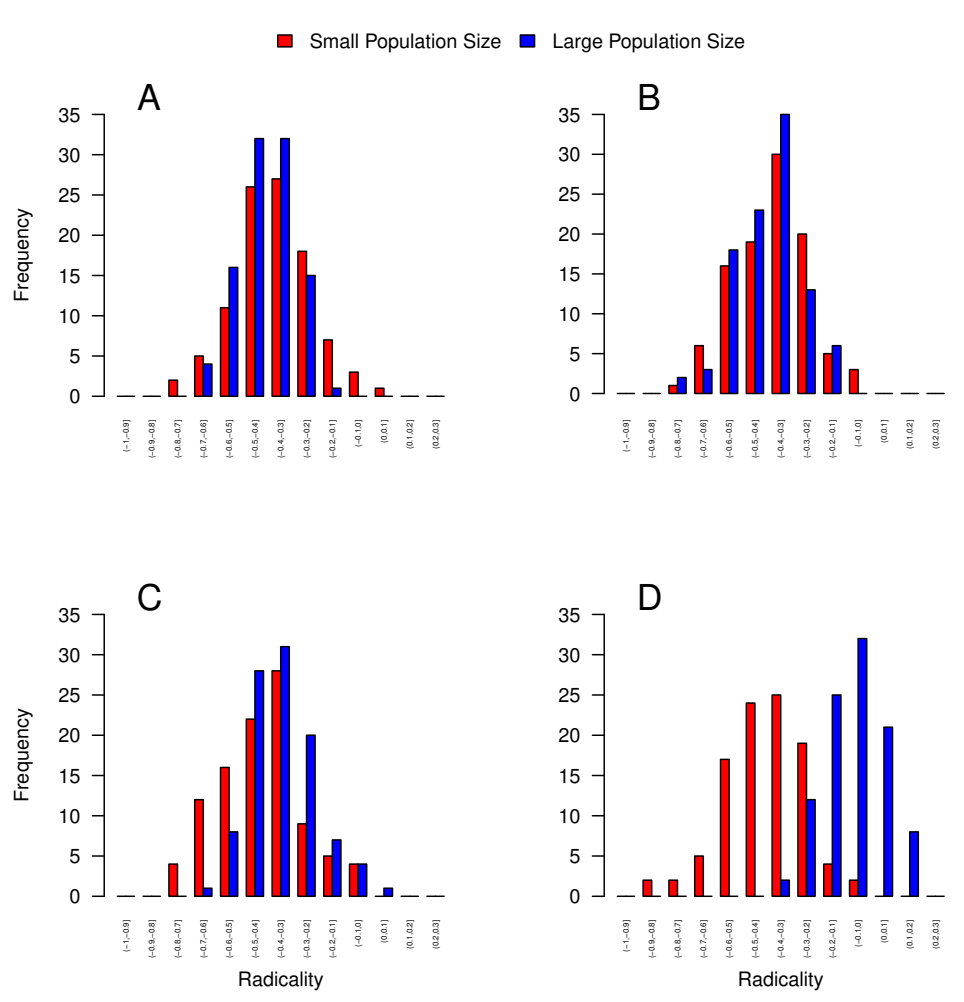

Figure 4.3: Inferences of differences in substitution radicality from simulated datasets, with smaller population sizes in red and larger population sizes in blue. When population size difference is nonexistent (panel A) or small (panel $\mathrm{B})$, there is no discernible difference in radicality between species. With larger population size differences of 100-fold (panel C) and 500-fold (panel D), small population-size species tend to have more radical substitutions. 


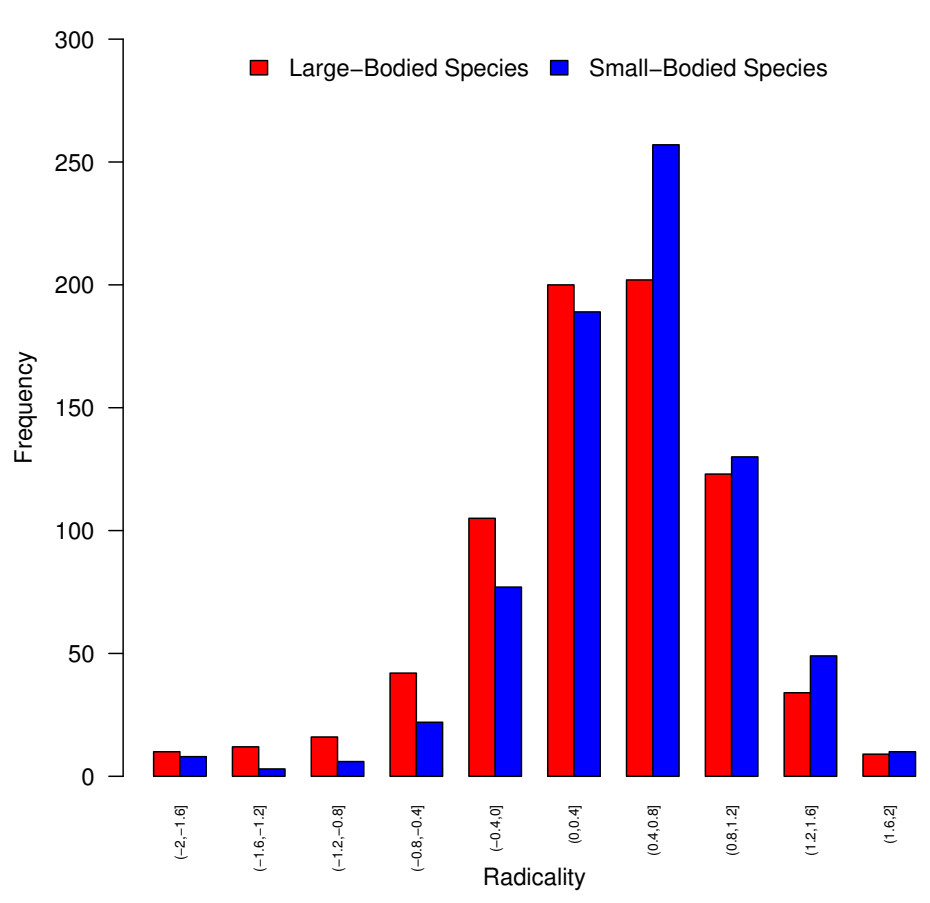

Figure 4.4: Radicality of average substitution for large vs. small-bodied species. More genes showed in large-bodied species had negative scores (radical substitutions) than small-bodied species.

simulated data for a 100-fold difference in population size. The 100-fold difference appears to correlate well with known effective population sizes in our dataset, as human $\mathrm{N}_{\mathrm{e}}$ is thought to be approximately 10,000 (Yu et al., 2004), and mice, for example, have an $\mathrm{N}_{\mathrm{e}}$ as high as 700,000 (Phifer-Rixey et al., 2012), a 70-fold difference. In performing 1000 bootstrap replicates in which species were randomly assigned into groups instead of being partitioned based on body size, a more extreme result was observed only 7 times, giving a p-value of 0.007 . Qualitatively similar results were found using PAM100, BLOSUM35, and BLOSUM62 matrices, indicating that this methodology is robust with several types of scoring matrix. 
To confirm our findings, we examined covariation between body size and the ratio of radical to conservative amino acid substitutions $(\mathrm{Kr} / \mathrm{Kc})$ using CoEvol, which jointly estimates both molecular and phenotypic characters along a tree using Markov Chain Monte Carlo techniques. Correlations can then be uncovered using the posterior probability estimates of positive or negative covariance between molecular and phenotypic traits of interest. Under the Polarity-Volume model, in which any substitution that changes the polarity or volume of an amino acid is considered radical, there was a significant enrichment in positive correlation between $\mathrm{Kr} / \mathrm{Kc}$ and body size (Figure 4.5). This distribution is significantly different from either a normal distribution or a uniform distribution centered at 0 (1000 bootstrap replicates for each, p-value $=0)$. Results showing the same trend with models based on charge, solely polarity, and solely volume yielded extremely similar distributions.

Finally, we investigated differences in the ratio of non-synonymous to synonymous substitutions for large and small bodied species using branch models in PAML. For 212 of 780 genes investigated, having a different $\omega$ for the two groups yielded a model with a significantly better fit. Within these 212 genes, the average $\omega$ for large body sizes was 0.189 , while small body sizes had an average $\omega$ of 0.133 (Figure 4.6), and 181 of the 212 genes $(85.4 \%$ ) had a higher value of $\omega$ for larger-bodied species. This difference was significant (Mann-Whitney U Test, $\mathrm{p}=5.8 \mathrm{x} 10-6)$. When all genes tested were considered, average for large-bodied species was 0.154 , while the average $\omega$ for small-bodied species remained 0.133. This difference was also significant (MannWhitney U Test, $\mathrm{p}=0.0045)$. 


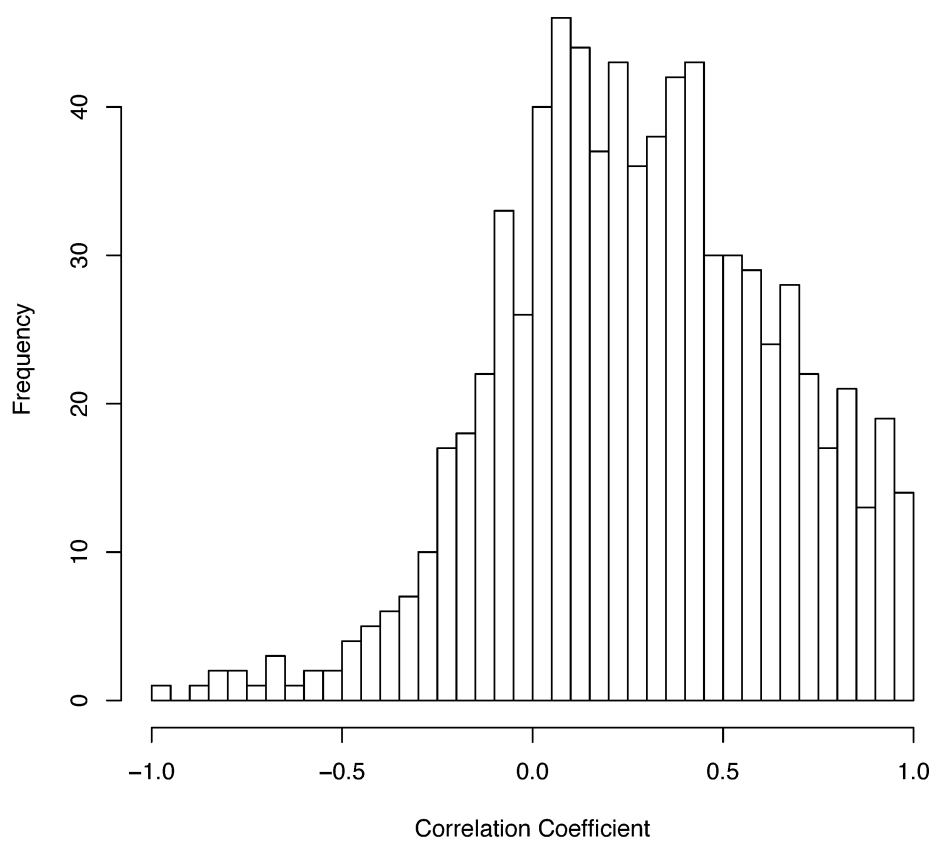

Figure 4.5: Correlation coefficients for a relationship between body size and $\mathrm{Kr} / \mathrm{Kc}$ for the polarity-volume model. Far more genes than would be expected under a null model have positive correlation coefficients. 


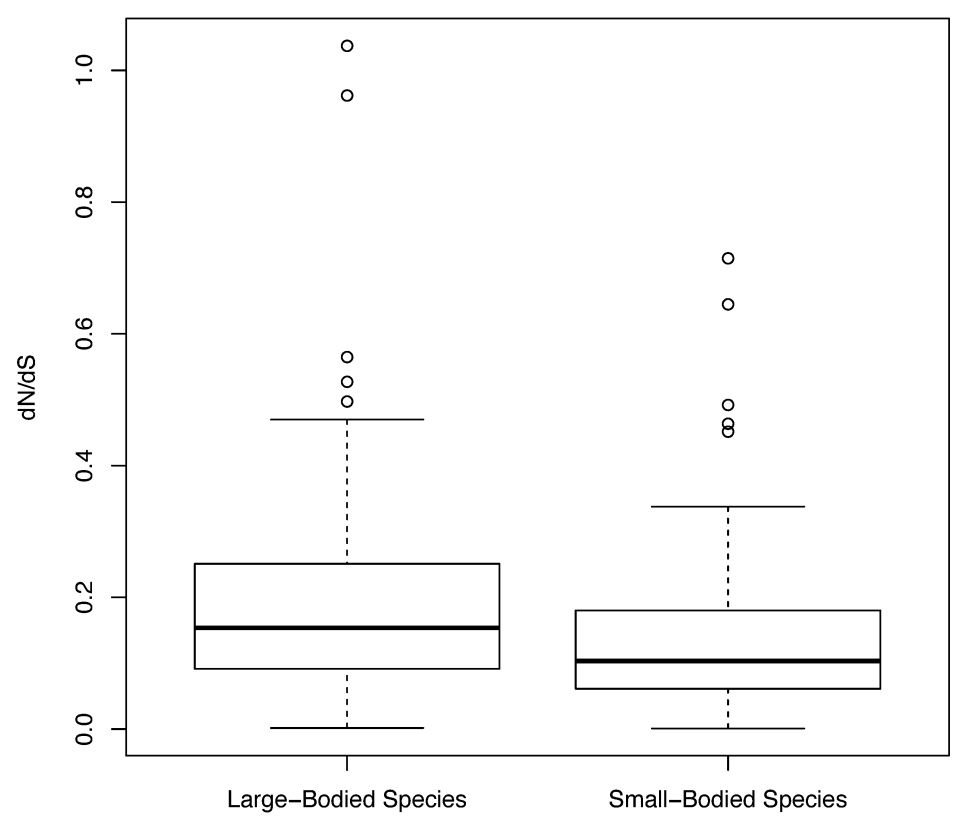

Figure 4.6: Boxplot of $\omega$ for each gene in which the three- $\omega$ model was a significant improvement over the null model. Large-bodied species show a significantly higher average $\omega$ than small-bodied species. 


\subsection{Discussion}

Effective population size is an important factor for many aspects of molecular evolution, particularly when considering the nearly neutral theory. Many important aspects of genome architecture and selection can be explained by the varying strength of selection with different effective population sizes. For example, the compact genomes of bacteria may be able to be explained by the fact that larger genomes are weakly selected against. Adding genetic material means more time spent on DNA replication, which will slow growth rates, and therefore decrease fitness. The magnitude of this effect is small, but due to the extremely large effective population size of bacteria, their genomes remain small. The same selective pressure is seen in higher order organisms, but due to their lower effective population sizes, the selection against larger genomes is not strong enough to be seen, leading to the creation of introns and large intergenic regions (Lynch and Conery, 2003). The strengths of positive and purifying selection vary widely across species, with even relatively small differences in $\mathrm{N}_{\mathrm{e}}$ showing notable effects on the strength of selection (Cagan et al., 2016). By taking advantage of new genomic resources and a novel substitution mapping-based approach to quantify the radicality of amino acid changes (and therefore purifying selection) we have shown that larger-bodied species tend to have more radical amino acid changes occur than small-bodied species. In addition, large-bodied species have higher ratios of non-synonymous to synonymous substitutions, presumably due higher rates of slightly deleterious non-synonymous substitutions. While the supply of synonymous (and therefore neutral) mutations will be higher in small-bodied species, the rate of fixation of neutral mutations will be the same in large and small-bodied species as the probability of fixation for a neutral mutation is proportional to $1 / N_{e}$, meaning that any variation in the ratio of non-synonymous to synonymous substitutions should be 
due to variation in non-synonymous substitutions.

Broadly speaking, these results are consistent with nearly neutral theory. Under near-neutrality, we expect that changes with slightly deleterious effects will be efficiently acted on by purifying selection in small-bodied species, while in large-bodied species the effect of these mutations will be too small to be acted on by selection. Previous work has shown that purifying selection does tend to select for more conservative amino acid changes (Smith, 2003).

An alternative explanation for our results could be that adaptive selection is the dominant selective force. The rate of adaptive evolution is thought to be independent of $\mathrm{N}_{\mathrm{e}}$ (Galtier, 2016), but the proportion of adaptive substitutions is higher in large $\mathrm{N}_{\mathrm{e}}$ species due to the stronger effect of drift (and therefore more slightly deleterious mutations) in small $\mathrm{N}_{\mathrm{e}}$ species. If this is the case, we can infer that adaptive substitutions may tend to be more conservative than those that are nearly neutral, as has previously been suggested (Nielsen and Huelsenbeck, 2002).

While the trend of more radical amino acid substitutions occurring in largerbodied mammals holds true with every methodology tested here, the trend does not hold perfectly at the species level. In particular, the Megabat (Pteropus vampyrus average radicality $=0.74$ ) and Black Flying Fox (Pteropus alecto - average radicality $=0.89)$ tend to undergo more radical substitutions than all other small-bodied species and many large-bodied species (average small body radicality $=1.10$, average large body radicality $=1.03)$. It is likely that the correlation between body mass and effective population size is not perfect. The entire clade containing bats within our tree tends to undergo more radical substitutions than would be expected for such small bodied species. Within the clade, the trend of larger-bodied species being more radical than smaller-bodied holds, as $P$. vampyrus and $P$. alecto (the two largest species in the clade) have more radical substitutions than other bats. It appears 
possible that bat life histories are sufficiently unique that their population sizes may be much smaller than would be anticipated based on their body size alone, but that larger-bodied bats still have smaller population sizes than small-bodied bats.

Substitution mapping-based approaches to the study of molecular evolution are promising and largely untapped until now. Though substitution mappings inferred through Bayesian techniques similar to those used in this study have existed since the early 2000s (Nielsen, 2002), molecular evolution studies of the mappings are relatively rare. As shown here, mappings are able to show qualitatively similar results to more established model-based techniques, but offer notable advantages over these approaches, in particular their speed and lack of need for complex models, as well as easy significance testing using predictive distributions (Nielsen and Huelsenbeck, 2002; Bollback, 2005; Romiguier et al., 2012).

The greatest advantage of substitution mapping-based approaches is their speed. Mappings can be generated in at most a few minutes for all genes used in this study, and could be sped up further through use of models other than the CAT-GTR used here. When tested, both the GTR and JTT models implemented in PhyloBayes MPI provided extremely similar results with a speedup of approximate 5x. Reading through the mappings is also quick, and could be further optimized as necessary. When comparing this to the analogous process of estimating $\omega$ for each gene using PAML, the speedup is clear; many genes take upwards of an hour of computation time, making analysis for datasets as large as this slow. This speedup makes mappingbased approaches attractive, particularly for exploratory data analysis where speed is a concern.

Related to the speedup of using mapping-based approaches to molecular evolution is the fact that using the evaluation of the mappings themselves is non-parametric, requiring no sophisticated models. To assess amino acid substitution radicality as was 
done here, each observed substitution was simply scored and these scores averaged. Similarly, a dN/dS ratio could be found by counting the number of non-synonymous and synonymous substitutions found in a tree; if one wished to, for example, find $\mathrm{dN} / \mathrm{dS}$ for each clade in a tree, the substitutions in those clades could also simply be counted, whereas a similar analysis in PAML would necessitate the use of an extremely parameter-rich model and take 60 times longer (Romiguier et al., 2012; Lemey et al., 2012). Many phenomena in molecular evolution can be examined in this simple and intuitive way, and mapping approaches also offer the opportunity to create posterior predictive p-values based on a comparison of the observed changes to the changes observed in a null distribution in which the same model is used, but the mappings in this distribution are not constrained to the data (Nielsen and Huelsenbeck, 2002; Bollback, 2005).

Though the non-parametric nature of the mappings used here certainly has advantages, there are also limitations to be considered. In particular, because analysis of the the mappings is non-parametric, quirks of the data that appear to show trends may be misleading. As an example, consider the case of assigning radicality to amino acid substitutions. Some amino acids, such as tryptophan, are highly dissimilar from essentially all other amino acids. Should one of our clades of interest have a tryptophan inferred at the base of the clade, almost any substitution that occurs will be considered to be quite radical, even though it may be one of the least radical substitutions available to tryptophan. Conversely, an alanine residue has few substitutions available that are negatively scored, meaning that clades with an alanine inferred at the base will be biased towards substitutions that appear to be quite conservative. We do not believe this to be the case here, both due to our large sample size (more than 400000 amino acid positions) and the fact that our clades of interest are spread across the entirety of the tree, instead of both large-bodied and small-bodied species 
being monophyletic. Additionally, due to the fact that we sample many draws from the posterior, and therefore many possible mappings, effects such as should be minimized. Nonetheless, awareness of the assumptions of mapping-based approaches is a key element of their use. Another way of mitigating these effects would be to develop more refined measurements of amino acid radicality. While the PAM and BLOSUM matrices used here were successful, they were not created with scoring of radicality in mind. Perhaps the best way to refine scoring of radicality would be to use an approach that is more grounded in the biochemical characteristics of the amino acids in question. This way (as has previously been done), amino acids could be grouped based on characteristics such as polarity, volume, or charge, and scored based on the number of characteristics that change.

Here, we have used a novel computational approach based on mapping mutations onto a phylogeny and scoring the radicality of these substitutions on previously unused genomic datasets in order to test whether or not the strength of purifying selection varies with population size, as would be expected under the nearly neutral theory. We find that our approach supports the nearly neutral theory, and is supported by similar results using more established techniques. 


\section{Chapter 5}

\section{Conclusions}

Substitution mappings have found less use than might be expected since first proposed by Nielsen (2002). Here, I have tested the usefulness of substitution mappings in two scenarios: detection of coevolving residues, and scoring of radicality for amino acid substitutions. Additionally, I have created a simulator, COMPASS, that is more flexible (to my knowledge) than any others currently available and is able to show entire substitution histories on a site-by-site basis; this facilitates studies that make use of mappings by giving true mappings for simulated data.

Using MI contained in substitution mappings tended to perform poorly, which was shown to be due to a lack of accuracy in substitution mappings for sites that were coevolving. This makes development of software that can produce more accurate mappings an obvious area for future work. Alternatively, given the relatively consistent error in the MI values for coevolving residues, it may be possible to develop a correction that makes the values of MI, and therefore coevolutionary inferences, more accurate.

On the other hand, scoring the radicality of amino acid substitutions seems to work quite well. For both simulated and real data, expected results can be reliably recovered using substitution mappings. The disparity between the success of the two 
studies can be explained by the fact that the algorithm for coevolution detection required not only correct transitions between states, but also correct timings for transitions between states, whereas scoring amino acid radicality requires only the former.

Based on these results, it appears that substitution mappings could see broad use in areas that require transitions but not exact timings. Current areas of application include calculations of dN/dS ratios, (Jobson et al., 2010; Romiguier et al., 2012, 2013), and inference of positively selected sites (Nielsen and Huelsenbeck, 2002; Lemey et al., 2012), but other countable phenomena in molecular evolution have the potential to be examined by substitution mappings. Due to the speed of inferring and reading through mappings, mapping-based approaches could have the potential to take the place of likelihood-based approaches such as those implemented in PAML (Romiguier et al., 2012). This is particularly true for large genomic datasets where speed is essential, which are likely to become more common in coming years.

In sum, substitution mappings are a promising avenue of research for studying processes in molecular evolution. 


\section{Bibliography}

Ackerman, S. H., Tillier, E. R., and Gatti, D. L. (2012). Accurate Simulation and Detection of Coevolution Signals in Multiple Sequence Alignments. PLoS ONE, $7(10)$

Adey, N. B., Tollefsbol, T. O., Sparks, A. B., Edgell, M. H., and Hutchison, C. A. (1994). Molecular resurrection of an extinct ancestral promoter for mouse L1. Proceedings of the National Academy of Sciences, 91(4), 1569-1573.

Ashkenazy, H., Penn, O., Doron-Faigenboim, A., Cohen, O., Cannarozzi, G., Zomer, O., and Pupko, T. (2012). FastML: A web server for probabilistic reconstruction of ancestral sequences. Nucleic Acids Research, 40(W1), 580-584.

Baker, F. N. and Porollo, A. (2016). CoeViz: a web-based tool for coevolution analysis of protein residues. BMC Bioinformatics, 17(1), 119.

Blanchette, M. (2004). Aligning Multiple Genomic Sequences With the Threaded Blockset Aligner. Genome Research, 14(4), 708-715.

Bollback, J. P. (2005). Posterior Mapping and Posterior Predictive Distributions. Statistical Methods in Molecular Evolution, pages 439-462.

Cagan, A., Theunert, C., Laayouni, H., Santpere, G., Pybus, M., Casals, F., Prüfer, K., Navarro, A., Marques-Bonet, T., Bertranpetit, J., and Andrés, A. M. (2016). 
Natural Selection in the Great Apes. Molecular Biology and Evolution, 33(12), msw215.

Collins, T. M., Wimberger, P. H., and Naylor, G. J. P. (1994). Compositional Bias, Character-State Bias, and Character-State Reconstruction Using Parsimony. Systematic Biology, 43(4), 482-496.

DeLange, R., Fambrough, D., Smith, E., and Bonner, J. (1969). Calf and pea histone IV. Journal of Biological Chemistry, 244(20), 5669-79.

Dib, L., Silvestro, D., and Salamin, N. (2014). Evolutionary footprint of coevolving positions in genes. Bioinformatics, 30(9), 1241-1249.

Dimmic, M. W., Hubisz, M. J., Bustamante, C. D., and Nielsen, R. (2005). Detecting coevolving amino acid sites using Bayesian mutational mapping. Bioinformatics, 21(SUPPL. 1), 126-135.

Dunn, S. D., Wahl, L. M., and Gloor, G. B. (2008). Mutual information without the influence of phylogeny or entropy dramatically improves residue contact prediction. Bioinformatics, 24(3), 333-340.

Dutheil, J. and Galtier, N. (2007). Detecting groups of coevolving positions in a molecule: a clustering approach. BMC Evolutionary Biology, 7, 242.

Dutheil, J., Pupko, T., Jean-Marie, A., and Galtier, N. (2005). A model-based approach for detecting coevolving positions in a molecule. Molecular Biology and Evolution, 22(9), 1919-1928.

Dutheil, J. Y., Galtier, N., Romiguier, J., Douzery, E. J. P., Ranwez, V., and Boussau, B. (2012). Efficient selection of branch-specific models of sequence evolution. Molecular Biology and Evolution, 29(7), 1861-1874. 
Eyre-Walker, A. (1998). Problems with parsimony in sequences of biased base composition. Journal of Molecular Evolution, 47(6), 686-690.

Fisher, R. A. (1930). The distribution of gene ratios for rare mutations. Proceedings of the Royal Society of Edinburgh, 50(1930), 205-220.

Fletcher, W. and Yang, Z. (2009). INDELible: A flexible simulator of biological sequence evolution. Molecular Biology and Evolution, 26(8), 1879-1888.

Galtier, N. (2016). Adaptive Protein Evolution in Animals and the Effective Population Size Hypothesis. PLoS Genetics, 12(1), 1-23.

Gowri-Shankar, V. and Rattray, M. (2007). A reversible jump method for Bayesian phylogenetic inference with a nonhomogeneous substitution model. Molecular Biology and Evolution, 24(6), 1286-1299.

Hietpas, R. T., Jensen, J. D., and Bolon, D. N. A. (2011). Experimental illumination of a fitness landscape. Proceedings of the National Academy of Sciences, 108(19), $7896-7901$.

Hudson, W. H., Kossmann, B. R., Mitchelle, I., Vera, S. D., Chuo, S.-w., Weikum, E. R., Eick, G. N., Thornton, J. W., Ivanov, I. N., Kojetin, D. J., and Ortlund, E. A. (2015). Distal substitutions drive divergent DNA specificity among paralogous transcription factors through subdivision of conformational space. Proceedings of the National Academy of Sciences, 113(2), 1-6.

Huelsenbeck, J. P. and Bollback, J. P. (2001). Empirical and hierarchical Bayesian estimation of ancestral states. Systematic Biology, 50(3), 351-366.

Hughes, A. L. and Friedman, R. (2009). More radical amino acid replacements in 
primates than in rodents: Support for the evolutionary role of effective population size. Gene, 440(1-2), 50-56.

Hughes, A. L. and Nei, M. (1988). Pattern of nucleotide substitution at major histocompatibility complex class I loci reveals overdominant selection. Nature, 335(6186), 167-170.

Jermann, T., Opitz, J., Stackhouse, J., and Benner, S. (1995). Reconstructing the evolutionary history of the artiodactyl ribonuclease superfamily. Nature, 374, 5759.

Jobson, R. W., Nabholz, B., and Galtier, N. (2010). An evolutionary genome scan for longevity-related natural selection in mammals. Molecular Biology and Evolution, $27(4), 840-847$.

Jones, D. T., Buchan, D. W. A., Cozzetto, D., and Pontil, M. (2012). PSICOV: Precise structural contact prediction using sparse inverse covariance estimation on large multiple sequence alignments. Bioinformatics, 28(2), 184-190.

Jones, D. T., Singh, T., Kosciolek, T., and Tetchner, S. (2015). MetaPSICOV: Combining coevolution methods for accurate prediction of contacts and long range hydrogen bonding in proteins. Bioinformatics, 31(7), 999-1006.

Kimura, M. (1968). Evolutionary rate at the molecular level. Nature, 217(5129), $624-626$.

Kryazhimskiy, S., Dushoff, J., Bazykin, G. A., and Plotkin, J. B. (2011). Prevalence of Epistasis in the Evolution of Influenza A Surface Proteins. PLoS Genetics, 7(2), e1001301. 
Lanfear, R., Welch, J. J., and Bromham, L. (2010). Watching the clock: Studying variation in rates of molecular evolution between species. Trends in Ecology and Evolution, 25(9), 495-503.

Lartillot, N. (2013). Interaction between selection and biased gene conversion in mammalian protein-coding sequence evolution revealed by a phylogenetic covariance analysis. Molecular Biology and Evolution, 30(2), 356-368.

Lartillot, N. and Poujol, R. (2011). A phylogenetic model for investigating correlated evolution of substitution rates and continuous phenotypic characters. Molecular Biology and Evolution, 28(1), 729-744.

Lartillot, N., Lepage, T., and Blanquart, S. (2009). PhyloBayes 3: A Bayesian software package for phylogenetic reconstruction and molecular dating. Bioinformatics, 25(17), 2286-2288.

Lartillot, N., Rodrigue, N., Stubbs, D., and Richier, J. (2013). PhyloBayes MPI : Phylogenetic Reconstruction with Infinite Mixtures of Profiles in a Parallel Environment. Software for Systematics and Evolution, 62(4), 611-615.

Lee, H.-J., Rodrigue, N., and Thorne, J. L. (2015). Relaxing the Molecular Clock to Different Degrees for Different Substitution Types. Molecular biology and evolution, 32(8), 1948-1961.

Lee, H.-J., Kishino, H., Rodrigue, N., and Thorne, J. L. (2016). Grouping substitution types into different relaxed molecular clocks. Philosophical Transactions of the Royal Society of London B, 371, 20150141.

Lemey, P., Minin, V. N., Bielejec, F., Pond, S. L. K., and Suchard, M. A. (2012). A counting renaissance: Combining stochastic mapping and empirical Bayes to 
quickly detect amino acid sites under positive selection. Bioinformatics, 28(24), $3248-3256$.

Lynch, M. and Conery, J. S. (2003). The origins of genome complexity. Science, 302(5649), 1401-1404.

Maddison, W. P. (1995). Calculating the Probability Distributions of Ancestral States Reconstructed by Parsimony on Phylogenetic Trees. Systematic Biology, 44(4), $474-481$.

Martin, L. C., Gloor, G. B., Dunn, S. D., and Wahl, L. M. (2005). Using information theory to search for co-evolving residues in proteins. Bioinformatics, 21(22), 41164124.

Nabholz, B., Uwimana, N., and Lartillot, N. (2013). Reconstructing the phylogenetic history of long-term effective population size and life-history traits using patterns of amino acid replacement in mitochondrial genomes of mammals and birds. Genome Biology and Evolution, 5(7), 1273-1290.

Nielsen, R. (2002). Mapping mutations on phylogenies. Systematic Biology, 51(5), 729-39.

Nielsen, R. and Huelsenbeck, J. P. (2002). Detecting positively selected amino acid sites using posterior predictive P-values. Pacific Symposium on Biocomputing, $\mathbf{5 8 8 ,}$ $576-88$.

Ohta, T. (1972a). Evolutionary rate of cistrons and DNA divergence. Journal of Molecular Evolution, 1(2), 150-157.

Ohta, T. (1972b). Population Size and Rate of Evolution. Journal of Molecular Evolution, 1(4), 305-14. 
Ohta, T. (1973). Slightly deleterious mutant substitutions in evolution. Nature, 246(5428), 96-98.

Pauling, L., Zuckerkandl, E., Henriksen, T., and Lövstad, R. (1963). Molecular "Restoration Studies" of Extinct Forms of Life. Acta Chemica Scandinavica, 17 supl., $9-16$.

Phifer-Rixey, M., Bonhomme, F., Boursot, P., Churchill, G. A., Piálek, J., Tucker, P. K., and Nachman, M. W. (2012). Adaptive evolution and effective population size in wild house mice. Molecular Biology and Evolution, 29(10), 2949-2955.

Pollard, K. S., Salama, S. R., King, B., Kern, A. D., Dreszer, T., Katzman, S., Siepel, A., Pedersen, J. S., Bejerano, G., Baertsch, R., Rosenbloom, K. R., Kent, J., and Haussler, D. (2006). Forces shaping the fastest evolving regions in the human genome. PLoS Genetics, 2(10), 1599-1611.

Popadin, K., Polishchuk, L. V., Mamirova, L., Knorre, D., and Gunbin, K. (2007). Accumulation of slightly deleterious mutations in mitochondrial protein-coding genes of large versus small mammals. Proceedings of the National Academy of Sciences, 104, 13390-13395.

Randall, R. N., Radford, C. E., Roof, K. A., Natarajan, D. K., and Gaucher, E. A. (2016). An experimental phylogeny to benchmark ancestral sequence reconstruction. Nature Communications, 7, 12847.

Romiguier, J., Figuet, E., Galtier, N., Douzery, E. J. P., Boussau, B., Dutheil, J. Y., and Ranwez, V. (2012). Fast and robust characterization of time-heterogeneous sequence evolutionary processes using substitution mapping. PLoS ONE, 7(3), 1-10. 
Romiguier, J., Ranwez, V., Douzery, E. J. P., and Galtier, N. (2013). Genomic evidence for large, long-lived ancestors to placental mammals. Molecular Biology and Evolution, 30(1), 5-13.

Sankoff, D. (1975). Minimal Mutation Trees of Sequences. SIAM Journal on Applied Mathematics, 28(1), 35-42.

Seemayer, S., Gruber, M., and Soding, J. (2014). CCMpred - Fast and precise prediction of protein residue-residue contacts from correlated mutations. Bioinformatics, 30(21), 3128-3130.

Shang, L., Xu, W., Ozer, S., and Gutell, R. R. (2012). Structural constraints identified with covariation analysis in ribosomal RNA. PLoS ONE, 7(6).

Siddiq, M. A., Loehlin, D. W., Montooth, K. L., and Thornton, J. W. (2017). Experimental test and refutation of a classic case of molecular adaptation in Drosophila melanogaster. Nature Ecology $\&$ Evolution, 1(January), 0025.

Smith, N. G. C. (2003). Are Radical and Conservative Substitution Rates Useful Statistics in Molecular Evolution? Journal of Molecular Evolution, 57(4), 467478.

Stewart, C. B. (1995). Active ancestral molecules. Nature, 374, 12-13.

Swanson, W. J. and Vacquier, V. D. (2002). The rapid evolution of reproductive proteins. Genetics, 3(February), 137-144.

Tacutu, R., Craig, T., Budovsky, A., Wuttke, D., Lehmann, G., Taranukha, D., Costa, J., Fraifeld, V. E., and De Magalhães, J. P. (2013). Human Ageing Genomic Resources: Integrated databases and tools for the biology and genetics of ageing. Nucleic Acids Research, 41(D1), 1027-1033. 
Talevich, E., Invergo, B. M., Cock, P. J. A., and Chapman, B. A. (2012). Bio.Phylo: a unified toolkit for processing, analyzing and visualizing phylogenetic trees in Biopython. BMC Bioinformatics, 13, 209.

Taylor, W. R., Jones, D. T., and Sadowski, M. I. (2012). Protein topology from predicted residue contacts. Protein Science, 21(2), 299-305.

Taylor, W. R., Hamilton, R. S., and Sadowski, M. I. (2013). Prediction of contacts from correlated sequence substitutions. Current Opinion in Structural Biology, $23(3), 473-479$.

Weber, C. C., Nabholz, B., Romiguier, J., and Ellegren, H. (2014). Kr/Kc but not $\mathrm{dN} / \mathrm{dS}$ correlates positively with body mass in birds, raising implications for inferring lineage-specific selection. Genome Biology, 15(542), 1-13.

Weigt, M., White, R., Szurmant, H., Hoch, J., and Hwa, T. (2009). Identification of direct residue contacts in protein-protein interaction by message passing. Proceedings of the National Academy of Sciences, 106(1), 67-72.

Wilson, C., Agafonov, R. V., Hoemberger, M., Kutter, S., Zorba, A., Halpin, J., Buosi, V., Otten, R., Waterman, D., Theobald, D. L., and Kern, D. (2014). Using ancient protein kinases to unravel a modern cancer drug's mechanism. Science, 5287(1995), 201-206.

Woolfit, M. and Bromham, L. (2005). Population size and molecular evolution on islands. Proceedings of The Royal Society of Biological Sciences, 272(1578), 22772282.

Wright, S. (1931). Evolution in mendelian populations. Bulletin of Mathematical Biology, 52(1-2), 241-295. 
Yang, Z. (2006). Computational Molecular Evolution. Oxford Series in Ecology and Evolution. Oxford University Press.

Yang, Z. (2007). PAML 4: Phylogenetic analysis by maximum likelihood. Molecular Biology and Evolution, 24(8), 1586-1591.

Yang, Z. and Nielsen, R. (2000). Estimating synonymous and nonsynonymous substitution rates under realistic evolutionary models. Molecular biology and evolution, 17(1), 32-43.

Yang, Z. and Nielsen, R. (2008). Mutation-selection models of codon substitution and their use to estimate selective strengths on codon usage. Molecular Biology and Evolution, 25(3), 568-579.

Yang, Z., Kumar, S., and Nei, M. (1995). A new method of inference of ancestral nucleotide and amino acid sequences. Genetics, 141(4), 1641-1650.

Yu, N., Jensen-Seaman, M. I., Chemnick, L., Ryder, O., and Li, W. H. (2004). Nucleotide Diversity in Gorillas. Genetics, 166(3), 1375-1383.

Zhang, J. and Nei, M. (1997). Accuracies of ancestral amino acid sequences inferred by the parsimony, likelihood, and distance methods. Journal of Molecular Evolution, 44(1 Supplement), 139-146. 


\section{Appendix A}

\section{Details on Coevolution Simulation}

To simulate nucleotide coevolution, a 16 by 16 rate matrix was created for use in COMPASS. The four possible Watson-Crick pairings (AT, TA, CG, and GC) were set as "preferred" nucleotide pairings. The coevolution strength determined equilibrium frequencies of all states, with each of the Watson-Crick pairs receiving one quarter of the coevolution strength as their frequency. Any remaining frequency was split between the 12 remaining pairs. For example, with a coevolution strength of 0.8 ,

the four Watson-Crick pairs would each have an equilibrium frequency of 0.2 , and all other pairs would have equilibrium frequencies of 0.0167 .

In the matrix itself, all transitions were allowed (i.e. double substitutions were allowed) based on the frequency of the "to" state, with equal exchangeabilities between all states. This setup results in the "preferred" pairs being together throughout evolutionary history in a proportion equal to their coevolution strength (with a coevolution strength of 0.8 , a paired site will be in a Watson-Crick pairing $80 \%$ of the time).

Amino acid simulations were carried out in much the same fashion as nucleotide substitutions. Four randomly chosen pairings were selected as "preferred" states, and 
the coevolution strength parameter split between them as previously described. Unlike the nucleotide simulations no double substitutions were allowed, as when double substitutions were allowed coevolution was detected, even at $0 \%$ coevolution strength with alignment or phylogenetic MI. Allowing only single substitutions alleviated this issue. 


\section{Appendix B}

\section{COMPASS Verification}

To simulate data for verifying exhangeabilities and nucleotide frequencies, 100 replicates were carried out for an alignment 150 nucleotides in length along a 130-species tree with 4 discrete gamma categories and an alpha value of 1 . PhyloBayes MPI was run for 1500 generations, taking the first 300 as burn-in under a CAT-GTR model with 4 discrete gamma categories in order to recover these values.

To simulate data to verify branch length accuracy, several trees were simulated using the PHASE package (Gowri-Shankar and Rattray, 2007), and a simulation under the same conditions used above was carried out 3 times for each tree. Total tree length was recovered using PhyloBayes MPI as described above.

To validate dN/dS data, data was simulated 100 times for an alignment 150 codons in length with a random $\mathrm{dN} / \mathrm{dS}$ value between 0 and 2 , with uniform rates across sites

on a 50-species tree. Estimates of $\mathrm{dN} / \mathrm{dS}$ were recovered using the codeml module of PAML 4.8 (Yang, 2007) under the M0 model. 